\title{
Comparison of Sonazoid contrast-enhanced ultrasound and gadolinium-ethoxybenzyl-diethylenetriamine pentaacetic acid MRI for the histological diagnosis of hepatocellular carcinoma
}

\author{
Feiqian Wang ${ }^{1,2}$, Kazushi Numata ${ }^{1}$, Masahiro Okada ${ }^{3}$, Makoto Chuma ${ }^{1}$, Hiromi Nihonmatsu ${ }^{1}$, \\ Satoshi Moriya ${ }^{1}$, Akito Nozaki ${ }^{1}$, Katsuaki Ogushi ${ }^{1}$, Wen Luo $^{4}$, Litao Ruan ${ }^{2}$, Masayuki Nakano ${ }^{5}$, \\ Masako Otani ${ }^{6}$, Yoshiaki Inayama ${ }^{6}$, Shin Maeda ${ }^{7}$ \\ ${ }^{1}$ Gastroenterological Center, Yokohama City University Medical Center, Yokohama, Kanagawa, Japan; ${ }^{2}$ Ultrasound Department, The First \\ Affiliated Hospital of Xi'an Jiaotong University, Xi'an, China; ${ }^{3}$ Department of Radiology, Nihon University School of Medicine, Itabashi-ku, Tokyo, \\ Japan; ${ }^{4}$ Department of Ultrasound, Xijing Hospital, Air Force Military Medical University, Xi'an, China; ${ }^{5}$ Tokyo Central Pathology Laboratory, \\ Utsukimachi, Hachioji, Japan; ${ }^{6}$ Division of Diagnostic Pathology, Yokohama City University Medical Center, Yokohama, Kanagawa, Japan; ${ }^{7}$ Division \\ of Gastroenterology, Yokohama City University Graduate School of Medicine, Yokohama, Kanagawa, Japan
}

Correspondence to: Kazushi Numata. Gastroenterological Center, Yokohama City University Medical Center, 4-57 Urafune-cho, Minami-ku, Yokohama, Kanagawa, 232-0024, Japan. Email: kz-numa@urahp.yokohama-cu.ac.jp.

Background: This study aimed to compare the value of Sonazoid contrast-enhanced ultrasound (SCEUS) with gadolinium-ethoxybenzyl-diethylenetriamine pentaacetic acid magnetic resonance imaging (EOB-MRI) for histological grading diagnosis, especially for early hepatocellular carcinoma (eHCC).

Methods: A total of 163 histopathologically confirmed HCC lesions were retrospectively collected, including 71 eHCCs (27 hypervascular, 44 non-hypervascular) and 92 advanced HCCs (adHCC) (73 hypervascular, 19 non-hypervascular). We performed SCEUS to evaluate the lesions' vascularity during the portal phase (PP) and the echogenicity during the post-vascular phase (PVP). EOB-MRI was used to determine the signal intensity between lesions and the surrounding liver parenchyma on unenhanced T1weighted images (pre-contrast ratio) in the hepatobiliary phase (HBP) (post-contrast ratio).

Results: For the PP and PVP of SCEUS (for all lesions), the pre-and post-contrast ratios of EOB-MRI (for all hypervascular lesions) showed statistical differences in the diagnosis of some (but not all) histological grades. For the diagnosis of eHCC, isoechogenicity in the PVP achieved the best diagnostic efficacy [area under the receiver operating characteristic curve $(A U C)=0.892]$. Whether used independently or in a combination of any form, all indicators failed to produce a higher diagnostic efficacy than PVP. Post- $(\geq 0.610)$ and pre-contrast ratios $(\geq 0.981)$ yielded acceptable diagnostic efficacy, with, respectively, accuracy levels of $69.3 \%$ and $75.5 \%$ and AUC values of 0.719 and 0.736 . For eHCC diagnosis, the post-contrast ratio $(\geq 0.625)$ and combined diagnosis using pre- $(\geq 0.907)$ and post-contrast ratios $(\geq 0.609)$ revealed the highest sensitivity (92.6\%) for hypervascular lesions and perfect specificity (100\%) for non-hypervascular lesions.

Conclusions: Unenhanced T1-weighted images and the HBP of EOB-MRI [regardless of the vascularity in the arterial phase (AP)], and the PP and PVP of SCEUS showed their value in the histological grading diagnosis of HCC. In particular, isoechogenicity in the PVP may have promising diagnostic utility for eHCC.

Keywords: Diagnosis; gadolinium-ethoxybenzyl-diethylenetriamine pentaacetic acid magnetic resonance imaging (EOB-MRI); hepatocellular carcinoma (HCC); histological grade; Sonazoid contrast-enhanced ultrasound (SCEUS)

Submitted May 22, 2020. Accepted for publication Jan 15, 2021.

doi: 10.21037/qims-20-685

View this article at: http://dx.doi.org/10.21037/qims-20-685 


\section{Introduction}

Worldwide, hepatocellular carcinoma (HCC) represents the sixth most common malignancy and the fourth most frequent cause of cancer-related death (1). In 2011, the incidence of HCC globally was 6.20 cases per 100,000 (2). In the current context of the serious epidemiological situation of HCC, conducting a further detailed histological grading diagnosis of HCC is worthwhile. This is because the histological staging system for HCC offers valuable and comprehensive information for prognosis and treatment and can even suggest a specific treatment for a given stage of HCC (3). Compared with advanced HCC (adHCC), patients with pathologically diagnosed early HCC (eHCC) lesions had much higher surgical cure rates, lower recurrence rates, and higher short-term and longterm survival rates (4-6). Even in small HCCs, which were believed to present an opportunity for minimally invasive therapies (7) and optimistic outcomes (8), eHCCs are quite different from adHCCs, as they have more favorable biological behaviors and prognosis than small hypervascular adHCCs (9). However, owing to their atypical characteristics, the diagnosis of eHCCs is thought to be missed more frequently than for adHCCs (10). It is therefore imperative to accurately detect and treat eHCCs in at-risk patients before they develop into adHCCs.

Histopathological examination of tumor biopsies or resection specimens has always been recognized as the gold standard for determining the histological grade of tumors. However, it is advantageous to explore non-invasive imaging approaches to obtain diagnostic information at the earliest possible time points (11). In recent years, the newly developed Sonazoid contrast-enhanced ultrasound (SCEUS) and gadolinium-ethoxybenzyl-diethylenetriamine pentaacetic acid magnetic resonance imaging (EOB-MRI) have become favored imaging methods. They are free of radiation, non-invasive, and repeatable. In particular, they can provide comprehensive information regarding vascularity and Kupffer cell function/hepatocyte function, allowing for improved diagnosis of HCC (12). Taking advantage of these multiple benefits, they are recommended in the guidelines as screening tools for HCC by the Japan Society of Hepatology (13). Moreover, the complementary application of the 2 imaging methods is preferred to improve diagnostic efficacy (14). In terms of histological grading diagnosis of HCC, on the one hand, several studies have confirmed the effectiveness of SCEUS and EOBMRI. Korenaga et al. reported an area under the receiver operating characteristic curve (AUC) as high as 0.996 for the post-vascular phase (PVP) of SCEUS in the detection of both moderate and poorly differentiated (diff.) HCCs from well diff. HCCs. Ohama et al. (15) demonstrated the usefulness of SCEUS in HCC differentiation according to the difference between well and poorly diff. HCC (P: 0.0010.013). Kogita et al. (16) and Kim et al. (17) confirmed the value of unenhanced T1-weighted and hepatobiliary phase (HBP) EOB-MRI in histological differentiation by comparing their signal intensity ratios (P: 0.001-0.01).

On the other hand, since these studies were heterogeneous in terms of their measurable indicators and histological classification standards, their reported results are incomparable. To the best of our knowledge, issues related to which imaging method is superior for histological grading diagnosis and whether the combined imaging patterns are more appropriate have not yet been fully explored. Investigations into a quantifiable diagnostic hallmark for imaging methods with definitive conclusions using relatively large sample sizes are still needed.

Our study was designed to clarify SCEUS and EOBMRI roles in detecting stepwise hepatocarcinogenesis by making a detailed comparison of their commonly used patterns between histological grades. By doing so, we further aimed to recommend a specific imaging pattern and method. Additionally, we propose optimal cutoff values for EOB-MRI as a reference standard for histological grading diagnosis.

\section{Methods}

\section{Patient enrollment}

During a period of 10.5 years, from September 2009 to February 2020, a total of 163 consecutive liver biopsy specimens from 126 patients were collected. All of these lesions were newly discovered and untreated, with a confirmed pathological diagnosis of HCC and definitive histopathological classification. The diagnosis of HCC was based on the Japan Society of Hepatology guidelines (13). For lesions with a typical imaging appearance of washin and wash-out, though the diagnosis of HCC could be made, we still performed a biopsy mainly with the purpose of histological grading diagnosis. Lesions with nonhypervascular or non-hyperintense appearance underwent a biopsy to make a definitive diagnosis. For all patients, grayscale US, SCEUS, and EOB-MRI examination of the liver were undertaken at least once within the month 


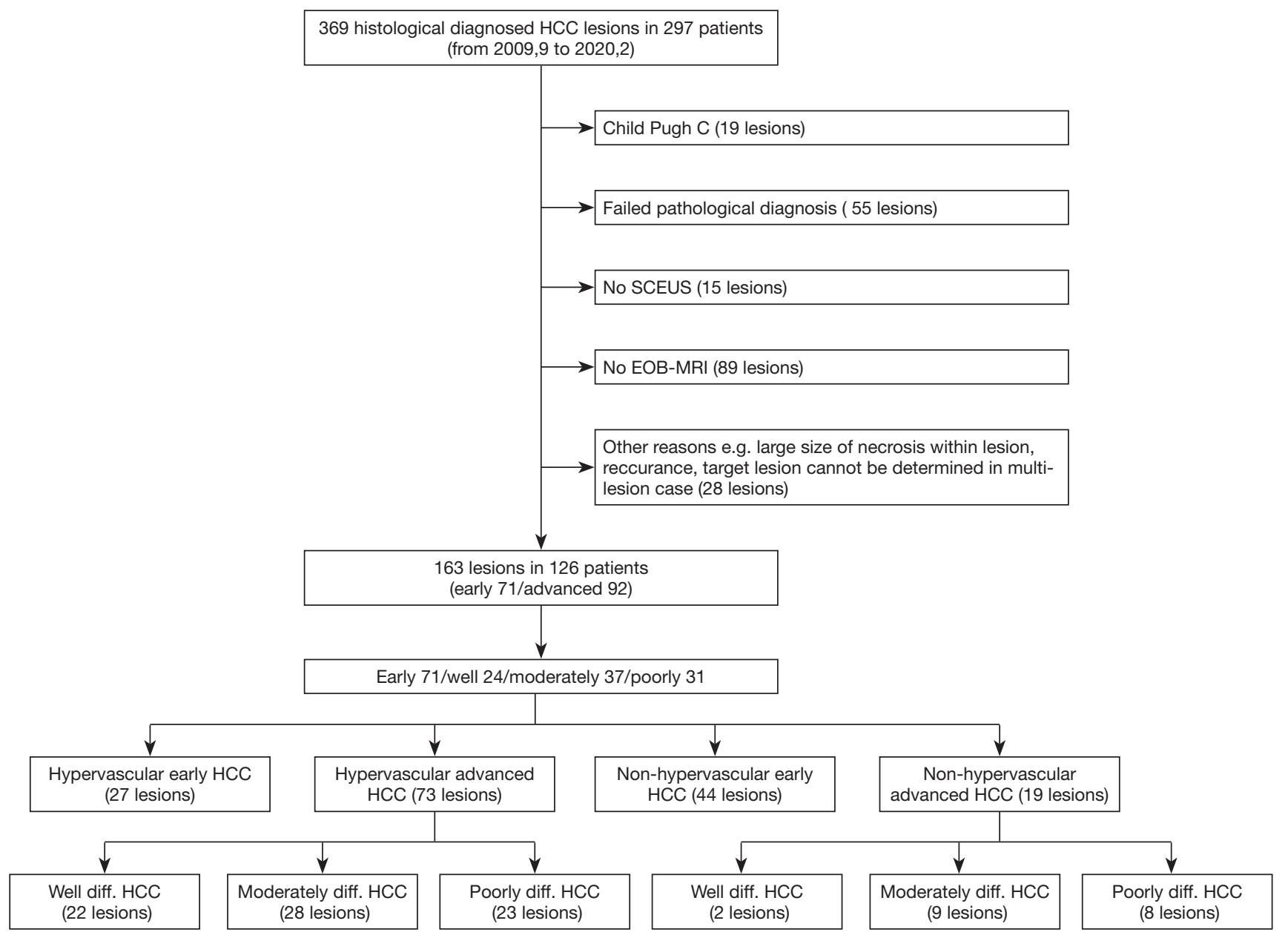

Figure 1 Flow chart depicting the selection of the study population. HCC, hepatocellular carcinoma; SCEUS, Sonazoid contrast-enhanced ultrasound; EOB-MRI, gadolinium-ethoxybenzyl-diethylenetriamine pentaacetic acid magnetic resonance imaging.

before the percutaneous US-guided liver biopsy. Clinical information (gender, age, etiology of cirrhosis, Child-Pugh classification, body mass index), imaging data, and histology reports were retrospectively collected from a review of the electronic medical recording system, the radiology database, and pathology records from our hospital, respectively. Our retrospective study design was approved by the institutional review board of Yokohama City University Medical Center (Number B180200054) and complied with the Declaration of Helsinki principles. The requirement for informed consent was waived. The exclusion criteria for the present study were as follows: (I) Child-Pugh class C liver cirrhosis (because of insufficient enhancement on MRI); (II) no SCEUS and/or EOB-MRI examination of the liver performed before the biopsy (within 1 month); (III) due to the insufficient sample size obtained by biopsy or the "no- hit' of the target lesion, a definite pathological diagnosis could not be obtained; (IV) systemic chemotherapy or targeted treatment performed before SCEUS and EOBMRI (which might have made the SCEUS and/or EOBMRI images atypical and thus difficult to diagnose); (V) a recurring lesion; and (VI) some large lesions with complex components, such as necrosis and/or hemorrhage (which is considered to create bias when evaluating the signal intensity of EOB-MRI and the vascularity of SCEUS).

The selection of the study population is presented in a flow chart (Figure 1).

\section{SCEUS protocol}

CEUS was performed using a US system LOGIQ E9 (GE Healthcare, Milwaukee, WI, USA) equipped with native 
tissue harmonic grayscale imaging and SCEUS function. A convex probe (frequency: 1-6 MHz) and a micro-convex probe (frequency: 2-5 MHz) were applied. We used a highfrequency linear probe with a frequency of $9 \mathrm{MHz}$ for scanning for lesions that were too superficial.

The size and position of the target lesion were initially observed by the grayscale US. Afterward, the SCEUS examination was undertaken. For US-undetectable lesions (isoechogenicity in grayscale US), with the assistance of fusion image technology, they could be accurately located in SCEUS with CT or MRI registration. An intravenous bolus injection of $0.2 \mathrm{~mL}$ perfluorobutane microbubbles (Sonazoid $^{\circledR}$, Daiichi Sankyo, Tokyo, Japan) was administered via the antecubital vein $0.2 \mathrm{~mL} / \mathrm{s}$ with a 24-gauge cannula, followed by injection of $2 \mathrm{~mL} 5 \%$ glucose. The lesions were observed and evaluated during 3 contrast phases, consisting of an arterial phase (AP) (10-50 s after initiation of injection), a portal phase (PP) (80-120 s after initiation of injection), and a PVP (10 min after initiation of the injection). In general, we used a low mechanical index mode $(0.21-0.28)$ as it preserves the integrity of microbubblestructure and allows real-time observation of the entire liver. In special cases, such as hyperechoic lesions (whose observation may be affected by the background B-mode) and deep-located lesions, we used high mechanical index (0.7-1.2) contrast imaging to accurately evaluate the presence or absence of defective areas during the PVP $(18,19)$.

\section{EOB-MRI protocol}

All EOB-MRI data were obtained by using a $1.5 \mathrm{~T}$ MR scanner (Avant; Siemens Medical System, Erlangen, Germany) with an 18-channel phased-array body coil and an integrated spine coil with 12 channels. Patients were asked to fast for 6-8 hours before the MR examination. The following examination steps were performed from our previously published study (20). Routine MR imaging sequences were high-spatial-resolution transverse T2-weighted imaging $\left(\mathrm{TR} / \mathrm{TE}=2,160 / 100 \mathrm{msec}\right.$; FOV $=433 \times 433 \mathrm{~mm}^{2}$; matrix size $=320 \times 288$; slice thickness $=6 \mathrm{~mm}$; flip angle $=160^{\circ}$ ), coronal T2-weighted imaging ( $\mathrm{TR} / \mathrm{TE}=1,000 / 96 \mathrm{~ms}$; FOV $=400 \times 400 \mathrm{~mm}^{2}$; matrix size $=320 \times 320$; slice thickness $=3 \mathrm{~mm}$; flip angle $=160^{\circ}$ ), and T1-weighted in- and opposed-phase imaging $\left(\mathrm{TR} / \mathrm{TE}=81 / 1.4 \mathrm{~ms}\right.$; FOV $=400 \times 325 \mathrm{~mm}^{2}$; matrix size $=352 \times 286$; slice thickness $=6 \mathrm{~mm}$; flip angle $=70^{\circ}$ ). After MR scanning with the unenhanced liver acquisition with volume acceleration and fat saturation, an EOB contrast agent (Primovist ${ }^{\circledR}$, Bayer Schering Pharma, Berlin, Germany) at a dose of $0.1 \mathrm{~mL} / \mathrm{kg}$ was injected at a rate of $1 \mathrm{~mL} / \mathrm{s}$ via a catheter in the antecubital vein. This was followed by $20 \mathrm{~mL}$ of saline flush administered at $1 \mathrm{~mL} / \mathrm{s}$. AP, PP, and late phase scanning were performed at 25-30, 70-85, and $180 \mathrm{~s}$ after the initiation of the contrast injection, respectively. HBP scanning was obtained at $20 \mathrm{~min}$ post-injection after the contrast injection initiation. Both unenhanced and HBP images were obtained as a T1-weighted high-resolution sequence of liver acquisition with volume acceleration (TR/ $\mathrm{TE}=6.2 / 3.15 \mathrm{~ms}$; flip angle, $20^{\circ}$; bandwidth, $260 \mathrm{~Hz} / \mathrm{pix}$; matrix, $166 \times 320)$ in a single breath hold $(18-20 \mathrm{~s})$.

\section{Imaging interpretation}

Compared with the adjacent non-tumorous regions, the liver parenchyma and vascularity patterns of lesions in SCEUS were classified as having hyper, iso, or hypovascularity when more than $50 \%$ of the lesion area corresponded to the above patterns. In EOB-MRI, all images were estimated in the transverse plane. The lesion's signal intensity and surrounding non-tumorous liver parenchyma were measured using an operator-defined region of interest on unenhanced T1-weighted and HBP images. The lesions were measured at the level of the largest diameter when they were displayed most clearly. The area without respiratory motion artifacts was chosen to be the surrounding area. Quantitative analysis of the precontrast ratio, post-contrast ratio, and $\mathrm{EOB}$ enhancement ratio was carried out with the following formulas taken from the literature (16):

Pre-contrast ratio = signal intensity of lesion/signal intensity of the non-tumorous regions in unenhanced T1weighted images.

Post-contrast ratio $=$ signal intensity of lesion/signal intensity of the non-tumorous regions in HBP images.

To assess the actual degree of tumor enhancement by EOB in comparison with the surrounding liver parenchyma, we also calculated the relative enhancement ratio of the lesions as follows (16):

$\mathrm{EOB}$ enhancement ratio $=$ post - contrast ratio/pre contrast ratio.

The schematic diagram of measurement is shown in Figure 2. Tumor vascularity was evaluated in the AP EOB-MRI images. The signal intensity of the tumorous area relative to the non-tumorous liver parenchyma was observed. A lesion's hypervascularity was defined as a hyperenhanced appearance compared with the surrounding 

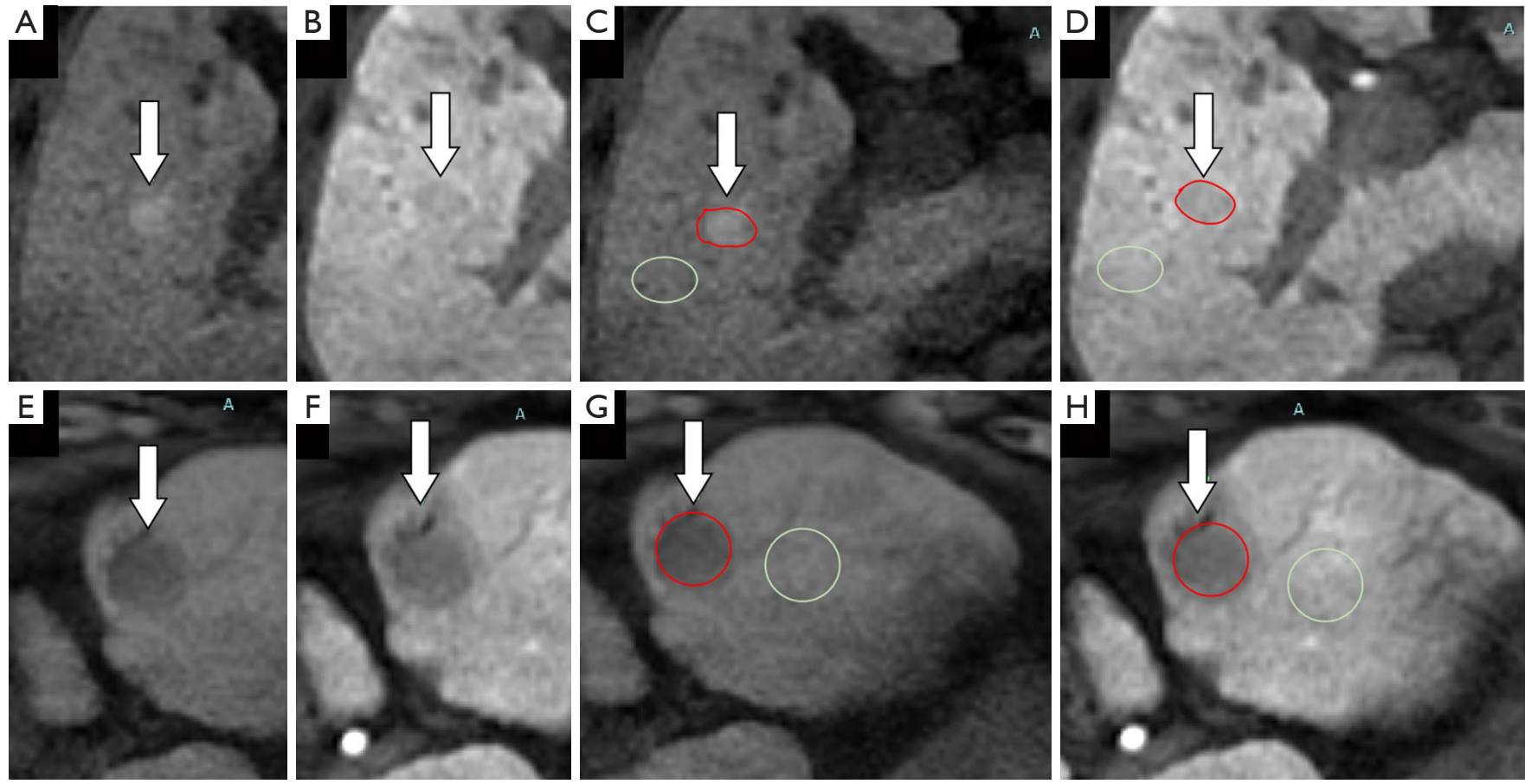

Figure 2 Schematic diagram of the quantitative measurement methods for the pre-contrast ratio, post-contrast ratio, and gadoliniumethoxybenzyl-diethylenetriamine pentaacetic acid (EOB) enhancement ratios. (A,B,C,D) and (E,F) are 2 different cases with characteristics of small size and unclear margin, respectively. Images (A,C,E,G) show the unenhanced T1-weighted images, while (B,D,F,H) show hepatobiliary phase $(\mathrm{HBP})$ images. In $(\mathrm{C}, \mathrm{D}, \mathrm{G}, \mathrm{H})$, the regions of interest of our target lesions are marked in red, and the surrounding nontumorous regions which were used as a reference are marked in green. In principle, the priority of reference should be given to uniform liver parenchyma without blood vessels with a similar size to the target lesions. (C,G) show unenhanced T1-weighted images, pre-contrast ratio = signal intensity of lesion (red area)/signal intensity of non-tumorous regions (green area). $(\mathrm{D}, \mathrm{H})$ show HBP images, post-contrast ratio = signal intensity of lesion (red area)/signal intensity of non-tumorous regions in the HBP image (green area). The EOB enhancement ratio = post-contrast ratio/pre-contrast ratio. The arrows indicate the target lesion.

liver parenchyma; otherwise, lesions were classified as nonhypervascular $(21,22)$.

Images obtained from EOB-MRI and SCEUS examinations were evaluated independently by 2 hepatologists (SCEUS: HN and KO; EOB-MRI: MC and $\mathrm{SM})$, who has worked in the field of HCC for 8 years and have more than 5 years of experience in liver imaging. For quantitative data (enhancement ratio in EOB-MRI), the average value was used for analysis. For qualitative data (echogenicity in SCEUS), any interpretation discrepancies were resolved by consensus with a third expert hepatologist (KN) with 20 years of HCC diagnosis and treatment experience. The operators were unaware of the final diagnosis, the patients' clinical conditions, and other radiological information.

\section{Histological diagnosis}

After confirming the HCC lesions' locations by grayscale US/SCEUS and EOB-MRI, a percutaneous fine-needle aspiration biopsy was performed under US/SCEUS guidance. Using a 21-gauge fine needle (Sonopsy ${ }^{\circledR}$, Hakko, Tokyo, Japan), we obtained no less than 2 samples from each lesion. Also, we obtained 1 sample of surrounding liver tissue as a negative control. All tissues were formalinfixed, paraffin-embedded, consecutively cut at the same thickness, and firstly stained with hematoxylin-eosin and silver impregnation, followed by Victoria blue, a cluster of differentiation 34, and cytokeratin 7. Histological diagnosis was performed by 2 board-certified pathologists (MO and YI). The diagnosis of HCC was made based on the 
Table 1 Baseline characteristics of patients/lesions enrolled according to histological grades

\begin{tabular}{|c|c|c|c|c|c|c|}
\hline Characteristics & Early $(n=71)$ & \multicolumn{3}{|c|}{ Advanced $(\mathrm{n}=92)$} & Total $(n=163)$ & $P$ value \\
\hline Age (mean \pm standard deviation, year) & $69.0 \pm 10.9$ & $70.5 \pm 9.1$ & $70.2 \pm 9.9$ & $73.3 \pm 10.6$ & $70.3 \pm 10.4$ & 0.306 \\
\hline Gender (female/male, n) & $14 / 57$ & $6 / 18$ & $11 / 26$ & $8 / 23$ & $39 / 124$ & 0.694 \\
\hline Child-Pugh classification (Class A/B, n) & $67 / 4$ & $19 / 5$ & $31 / 6$ & $27 / 4$ & $144 / 19$ & 0.154 \\
\hline BMI (mean \pm standard deviation, $\mathrm{kg} / \mathrm{m}^{2}$ ) & $22.8 \pm 4.1$ & $24.1 \pm 2.9$ & $23.5 \pm 3.6$ & $23.2 \pm 4.1$ & $23.2 \pm 3.8$ & 0.506 \\
\hline Size $(\leq 2 \mathrm{~cm} />2 \mathrm{~cm})$ & $63 / 8$ & $18 / 6$ & $28 / 9$ & $21 / 10$ & $130 / 33$ & 0.071 \\
\hline
\end{tabular}

${ }^{\dagger}$, here, others indicate alcohol abuse, nonalcoholic steatohepatitis, primary biliary cirrhosis and some unknown reasons. A few patients have two or more primary HCC related diseases simultaneously, so the number of the counts is larger than the number of patients.

criteria outlined by an International Working Party (23). The histopathological grades of HCC were taken from Edmondson and Steiner's classification (24) and the International Consensus Group (25), with a minor change according to our more than 10 years of clinical experience in HCC diagnosis. We classified HCCs roughly into eHCCs and adHCCs. In detail, adHCC was classified into 3 grades: well, moderately, and poorly diff. HCC.

\section{Statistical analysis}

As described earlier, the target patterns in this study were the pre-contrast ratio, post-contrast ratio, and $\mathrm{EOB}$ enhancement ratio in EOB-MRI, as well as the PP and PVP in SCEUS. Continuous variables that showed a normal distribution (age and post-contrast ratio) were compared with $t$-tests. For all other variables (tumor size, pre-contrast ratio, and $\mathrm{EOB}$ enhancement ratio) that showed a nonnormal distribution, we used the Mann-Whitney $U$ test to compare histological groups. Continuous variables are presented as the mean and standard deviation (normal distribution) or median and interquartile range (nonnormal distribution). Lesion size was presented as the mean and range. Categorical variables such as gender, etiology of HCC, and target patterns of SCEUS were compared using Fisher's exact test or the chi-squared test. Spearman correlation analysis was performed between the 2 imaging methods' target patterns and the 4 different histological grades. Similarly, we conducted difference (Fisher's exact test or the chi-squared test) and relationship (Spearman correlation) analyses of hypervascular lesions in terms of their detailed histological grades.

To evaluate the diagnostic efficacy of the target patterns for eHCC, we classified all lesions into eHCC and adHCC subgroups. The cutoff values for the target patterns in EOBMRI were obtained from receiver operating characteristic curve analysis. Using these cutoff values, we performed intergroup comparisons of histological grade using chisquared tests. The sensitivity, specificity, and accuracy of all target patterns for eHCC diagnosis were calculated. The differences in the target patterns in EOB-MRI between hypervascular and non-hypervascular eHCCs were calculated. All statistical analyses were performed using SPSS version 26.0 for Windows (SPSS Inc., Chicago, IL, USA). Two-tailed $\mathrm{P}<0.05$ was considered to be statistically significant.

\section{Results}

\section{Baseline characteristics}

Table 1 presents the baseline characteristics of the study population. The number of HCC patients affected by the hepatitis $\mathrm{C}$ virus $(\mathrm{HCV})$, hepatitis $\mathrm{B}$ virus (HBV), and other diseases (such as nonalcoholic steatohepatitis and primary biliary cirrhosis) was 108,25 , and 40 , respectively. The mean diameter of all lesions was $16.6 \mathrm{~mm}$ (range, $7-45 \mathrm{~mm}$ ). No significant differences in general characteristics (age, gender, etiology of HCC, body mass index, Child-Pugh classification, and lesion size) were observed between the 2 subgroups of histological grades $(\mathrm{P}>0.05$, Table 1$)$.

\section{Comparison of SCEUS and EOB-MRI enhancement patterns between 4 histological grades}

Apart from 1 case of poorly diff. HCC showing hyperenhancement, the remaining 162 cases in our study 
Table 2 The relationship between indicators and histology classification of $\mathrm{HCC}^{\dagger}$

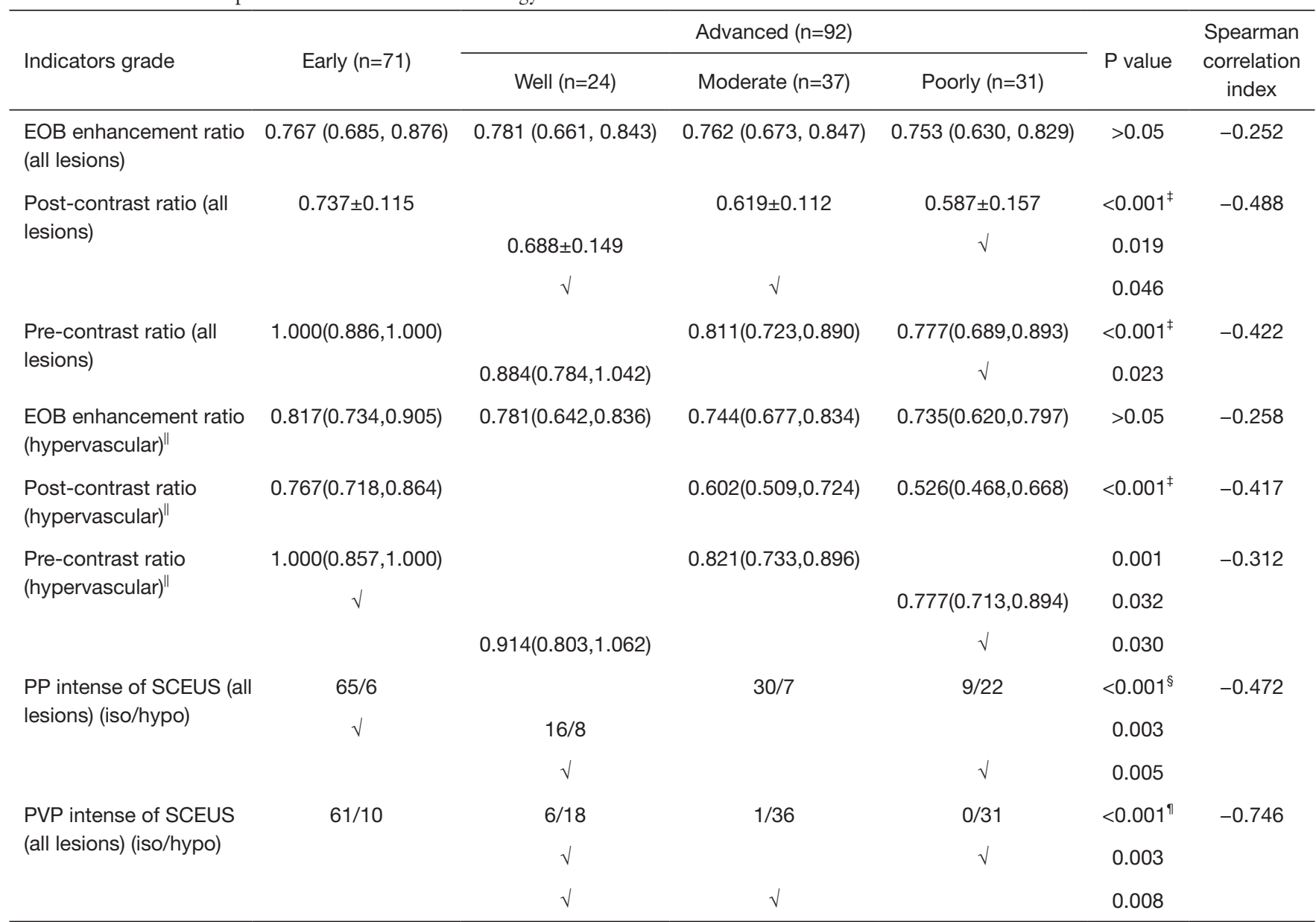

${ }^{\dagger}$, HCC, hepatocellular carcinoma; SCEUS, Sonazoid contrast enhanced ultrasound; EOB-MRI, gadolinium ethoxybenzyl diethylenetriamine pentaacetic acid magnetic resonance imaging; AP, arterial phase, PP, portal phase; PVP, post-vascular phase; AUC, area under the curve. ${ }^{\ddagger}$, here, the value means the comparison between early and well diff. HCC, early and poorly diff. HCC have a P value lower than 0.001 .

$\S$, here, the value means the comparison between poorly and eHCC, poorly and moderately diff. HCC have a P value lower than 0.001 .

$"$, here, the value means the comparison between early and any other diff. HCC all have a P value lower than 0.001 . ", for hypervascular lesions, the numbers of early, well, moderately, poorly diff. HCCs were 27, 22, 28 and 23.

exhibited a non-hyperenhanced appearance in the HBP of EOB-MRI. In terms of the number of lesions with a hyper-, iso-, or hypoenhanced appearance in the unenhanced T1weighted images, there were, respectively, 10, 30, and 31 eHCC lesions; 6, 1, and 17 well diff. HCC lesions; 2, 1, and 34 moderately diff. HCC lesions; and 1, 0 , and 30 poorly diff. HCC lesions. When each diagnostic indicator was analyzed between every 2 histological grades (Table 2), the vascularity of the $\mathrm{PP}$ and the echogenicity of the PVP in SCEUS, the signal intensity ratio in unenhanced T1-weighted images and the HBP of EOB-MRI were significantly different in some (but not all) histological grades. Specifically, all of the target patterns of EOB-MRI allowed clear differentiation of eHCC (Figure 3) from moderately and poorly diff. HCC. A post-contrast ratio further distinguished well diff. HCC (Figures 4,5) from moderately (Figure 6) and poorly (Figure 7) diff. HCC. The pre-contrast ratio, irrespective of the hypervascularity of all lesions, differed significantly between well diff. and poorly diff. HCC. Except for the comparison between moderately and poorly diff. HCC, a difference in the PVP of SCEUS was revealed between every 2 histological groups. Differences in the PP were observed between poorly diff. HCC and all other histological grades, as well as between eHCC and well diff. HCC. However, the EOB enhancement ratio was revealed to have no significance in 

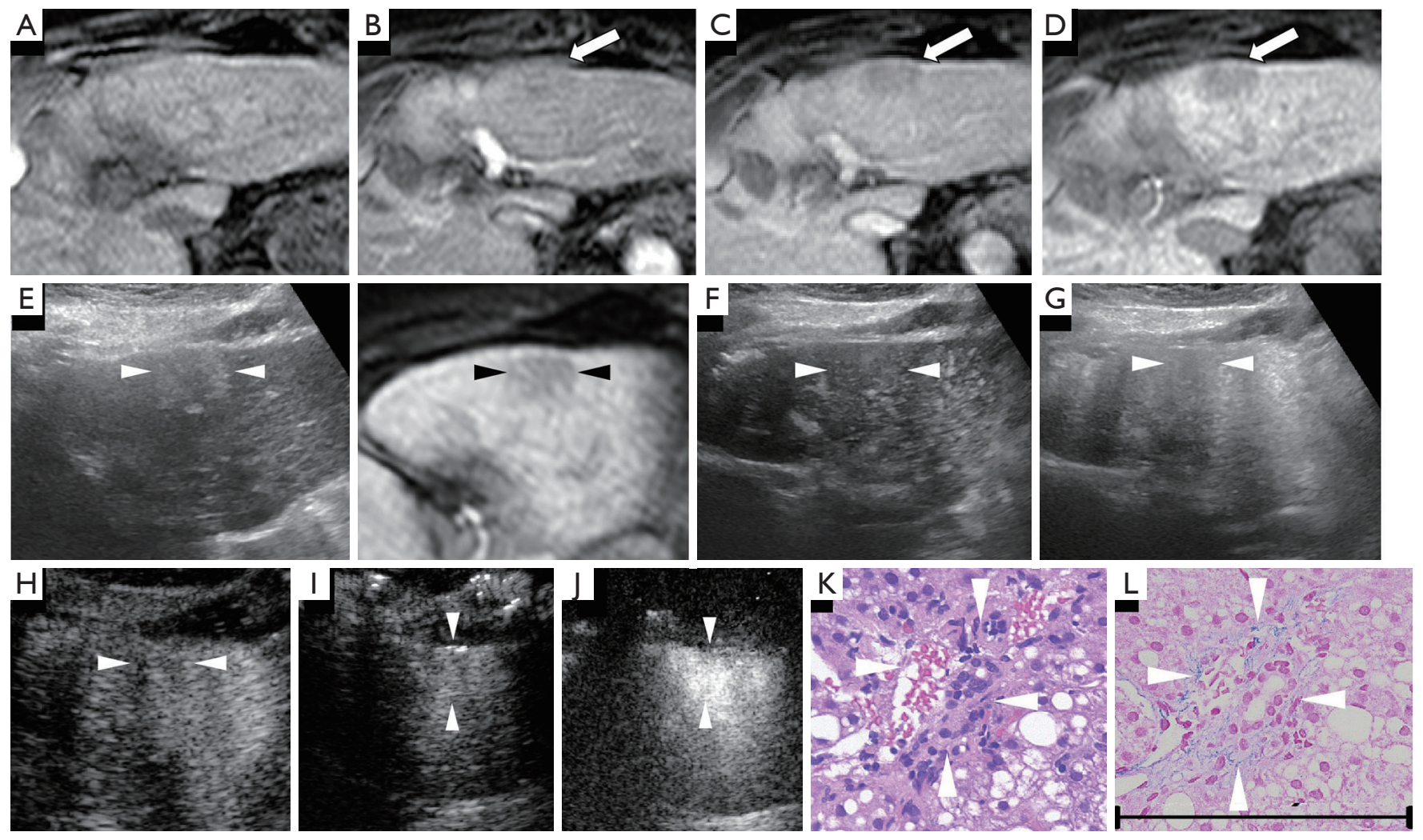

Figure 3 A case of an early hepatocellular carcinoma (HCC) lesion (maximum diameter, $25 \mathrm{~mm}$ ) in the subcapsular liver of segment III in an 88-year-old female patient with chronic hepatitis C-related disease. (A) Axial T1WI images on a plain scan show no lesion in the liver parenchyma. The pre-contrast ratio was 1.00. (B) On the arterial phase of gadolinium-ethoxybenzyl-diethylenetriamine pentaacetic acid magnetic resonance imaging (EOB-MRI) (25 seconds), the lesion is shown as slightly hypointense compared with surrounding background liver parenchyma. No intratumoral vessels are visible. (C) On the PP of EOB-MRI (85 seconds), the lesion is shown as hypointense compared with the surrounding background liver parenchyma. (D) Twenty minutes after EOB agent injection, the lesion appeared as a homogeneous hypointensity (arrow) on the hepatobiliary phase. The post-contrast ratio and EOB enhancement ratio were both 0.830 . (E) Fusion images combining grayscale ultrasound (US) (left side) and the hepatobiliary phase of EOB-MRI as a reference (right side) on a single screen. In the grayscale US, it shows the lesion as a slightly hyperechoic area with an unclear boundary in segment V. (F,G) Using low mechanical index contrast imaging, the arterial phase (G) and PP (H) of Sonazoid contrast-enhanced ultrasound (SCEUS) show a nonhypervascular lesion. (H,I) The post-vascular phase shows an isoechoic lesion (no washout) (H: 8 min, I: 11 min). (J) Using high mechanical index contrast imaging $(16 \mathrm{~min})$, the lesion in the post-vascular phase still had an isoechoic appearance. (K,L) Hematoxylin-eosin staining (K) shows a slightly increased cell density in the tumor area. Portal tract invasion (short white arrowheads), which is a unique finding in early HCC, was identified with hematoxylin-eosin staining $(\mathrm{K})$ and Victoria blue staining $(\mathrm{L})$. The arrows seen in (B,C) and the arrowheads in (E,F,G,H,I,J) indicate the margin of the lesion. The black scale's length in the lower side of this figure represents $200 \mathrm{microns}(\mu \mathrm{m})$ as a magnification reference for both $(\mathrm{K}, \mathrm{L})$. The tumor was histopathologically confirmed as early HCC.

the differential diagnosis of any histological grade, whether for hypervascular lesions or for all lesions.

\section{Correlation analysis}

The Spearman correlation analysis showed a negative correlation between all of the target patterns and the histological grade $(\mathrm{P}<0.05)$. The PVP of SCEUS had the best correlation, with a correlation index of 0.746 , suggesting a strong correlation between the PVP's isoechogenicity and the histological grade. The EOB enhancement ratio showed a very weak correlation with the histological grade, with correlation indexes of -0.312 and -0.252 in hypervascular lesions and all lesions, respectively. 

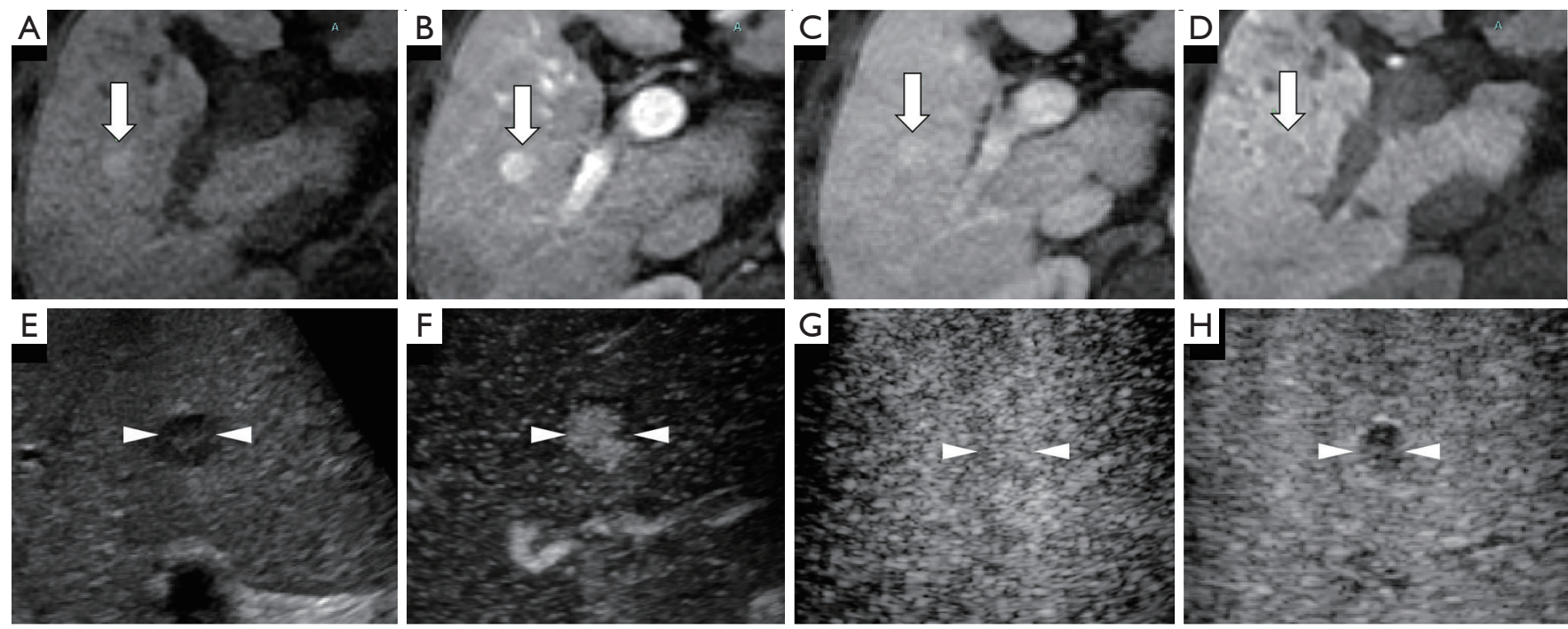

Figure 4 A case of a well-differentiated hepatocellular carcinoma (HCC) lesion (maximum diameter, $15 \mathrm{~mm}$ ) in segment V/VIII in a 56-year-old male patient with chronic hepatitis C-related disease. (A) Axial T1WI images on a plain scan show homogeneous hyperintensity in the tumor. The pre-contrast ratio was 1.199. (B,C) On the arterial phase (B, 25 seconds) and portal phase (C, 70 seconds) of gadoliniumethoxybenzyl-diethylenetriamine pentaacetic acid magnetic resonance imaging (EOB-MRI), the lesion is shown as slightly hyperintense compared with surrounding background liver parenchyma. (D) Twenty minutes after EOB agent injection, the lesion shows as a homogeneous hypointensity (arrow) on the hepatobiliary phase. The post-contrast ratio was 0.616 and the EOB enhancement ratio was 0.514. (E) Grayscale US shows a homogeneous hypo-echoic lesion with oval shape and clear margin. No halo is seen around the lesion. (F) Using low mechanical index contrast imaging, the arterial phase of Sonazoid contrast-enhanced ultrasound (SCEUS) shows hypervascular enhancement. $(\mathrm{G}, \mathrm{H})$ The lesion shows isovascularity in the portal phase $(\mathrm{G})$ and slightly hypoechoic $(\mathrm{H})$ in the post-vascular phase (late washout). The arrows seen in (A,B,C,D) and the arrowheads seen in (E,F,G,H) indicate the margin of the lesion.

Other target patterns showed a medium correlation ranging from -0.417 to -0.488 (Table 2).

\section{Analysis of the diagnostic efficacy of indicators for eHCC}

As seen in Table 3, for all lesions, the cutoff values for the pre-and post-contrast ratios obtained from the receiver operating characteristic curve analysis were 0.981 and 0.610 , respectively. In hypervascular lesions, the pre-and post-contrast ratios' cutoff values were 0.984 and 0.625 , respectively. In non-hypervascular lesions, the pre-and post-contrast ratios' cutoff values were 0.907 and 0.609 , respectively. These cutoff values were used as standards for eHCC diagnosis. For all lesions, among EOB-MRI's target patterns, the pre-contrast ratio (0.981) was relatively more efficient, yielding an accuracy of $75.5 \%$ and an AUC of 0.736 . However, among the target patterns of SCEUS, the PVP was relatively more efficient.

Furthermore, among all patterns, the PVP yielded the highest accuracy (89.6\%) and AUC value (0.892). The combined application of any target pattern could not achieve higher diagnostic efficacy than the independent application of PVP. For hypervascular lesions, the postcontrast ratio produced an acceptable diagnostic result, with an AUC of 0.650 and an accuracy of $73.7 \%$. The precontrast ratio for non-hypervascular lesions showed the best diagnostic efficacy among all target patterns in the EOBMRI examination groups. The accuracy and AUC were $82.5 \%$ and 0.860 , respectively. A combination of the preand post-contrast ratios could increase the specificity to $100 \%$ at the cost of a slightly lower AUC (0.830). Although there was no big difference in the AUC compared to all lesions, the sensitivity $(92.6 \%)$ obtained by the post-contrast ratio $(0.625)$ was the highest among all target patterns. Overall, the isoechoic appearance in the PVP performed best for eHCC diagnosis.

\section{The value of EOB-MRI in distinguishing hypervascular and non-hypervascular eHCCs}

There was no statistical difference in the pre-contrast ratio. However, the post-contrast ratio and EOB enhancement 

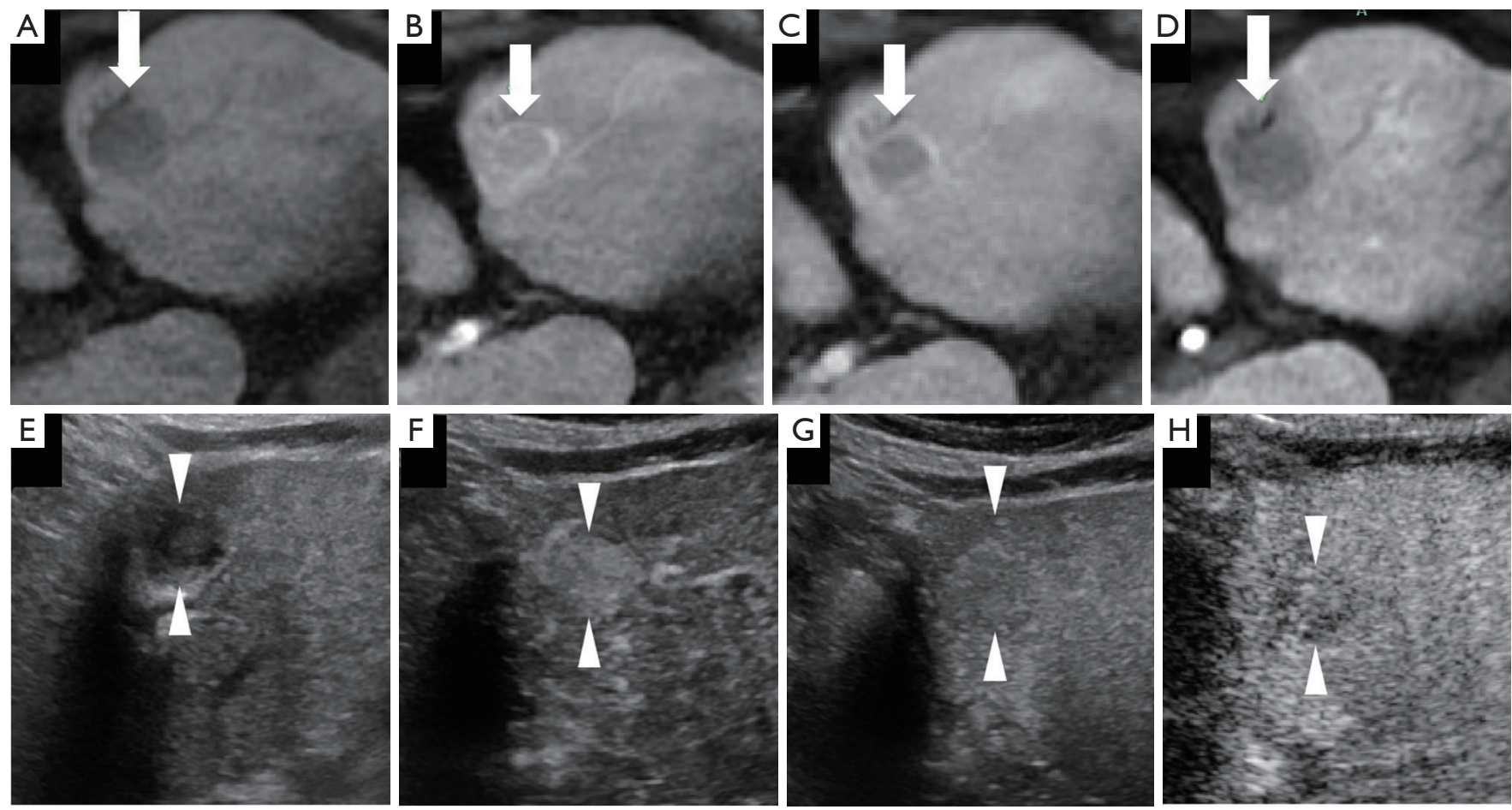

Figure 5 A lesion diagnosed as well-differentiated hepatocellular carcinoma (HCC) (maximum diameter, $20 \mathrm{~mm}$ ) in segment III was found in an 82-year-old male patient with chronic hepatitis C. (A) Axial T1WI image on a plain scan shows homogeneous hypointensity in the tumor. The pre-contrast ratio was 0.724 . (B) On the arterial phase of gadolinium-ethoxybenzyl-diethylenetriamine pentaacetic acid magnetic resonance imaging (EOB-MRI) (25 seconds), the lesion is shown as an isointensity, with significant branched peripheral blood vessels. (C) On the portal phase of EOB-MRI (70 seconds), the lesion is shown as a hyperintensity compared with the surrounding background liver parenchyma. (D) The lesion shows as a homogeneous hypointensity (arrow) on the hepatobiliary phase. The boundary is not very clear. The post-contrast ratio was 0.468 and the EOB enhancement ratio was 0.646. (E) The targeted lesion appears as a well-defined, homogeneous, non-hyperechoic lesion on grayscale ultrasound (US), without a surrounding halo. (F) Using low mechanical index contrast imaging, the arterial phase and the portal phase of Sonazoid contrast-enhanced ultrasound (SCEUS) show hypervascular and isovascular areas, respectively, with a homogeneous pattern. (G) During the post-vascular phase, the lesion appears as slightly hypoechoic. (H) After switching to high mechanical index contrast imaging, the lesion is shown more clearly as a defective area during the post-vascular phase. The arrows seen in $(A, B, C, D)$ and the arrowheads seen in $(E, F, G, H)$ indicate the margin of the lesion.

ratio was significantly different $(\mathrm{P}<0.05)$ (Table 4$)$. In general, non-hypervascular eHCCs showed a lower post-contrast ratio and $\mathrm{EOB}$ enhancement ratio than hypervascular eHCCs.

\section{Discussion}

Our research showed that the PP of SCEUS demonstrated efficacy in differentiating the histological grade of HCC. The contrast agent of SCEUS enters into the luminal compartment of blood vessels shortly after administration (27). Thus, the appearance of lesions in SCEUS can show intralesional blood flow changes and generate a so-called vascular image.
As hepatocarcinogenesis advances, portal flow begins to decrease while neovascularization initially develops. In eHCCs, this slight change in blood supply may not lead to an abnormal appearance of the lesions than the surrounding parenchyma. Therefore, in the PP of SCEUS, most HCC lesions show an isovascular appearance. According to the observations of the $\mathrm{PP}$ in our research, almost all eHCC lesions $(65 / 71,91.5 \%)$ had an isovascular appearance. With the histological development of HCC, the portal blood supply reduces or even reverses $(16,28)$. The change in blood flow is aggravated with the stepwise advancement of HCC. This was confirmed by our findings.

In the PP, the incidence of isovascularity in well $(16 / 24$, 

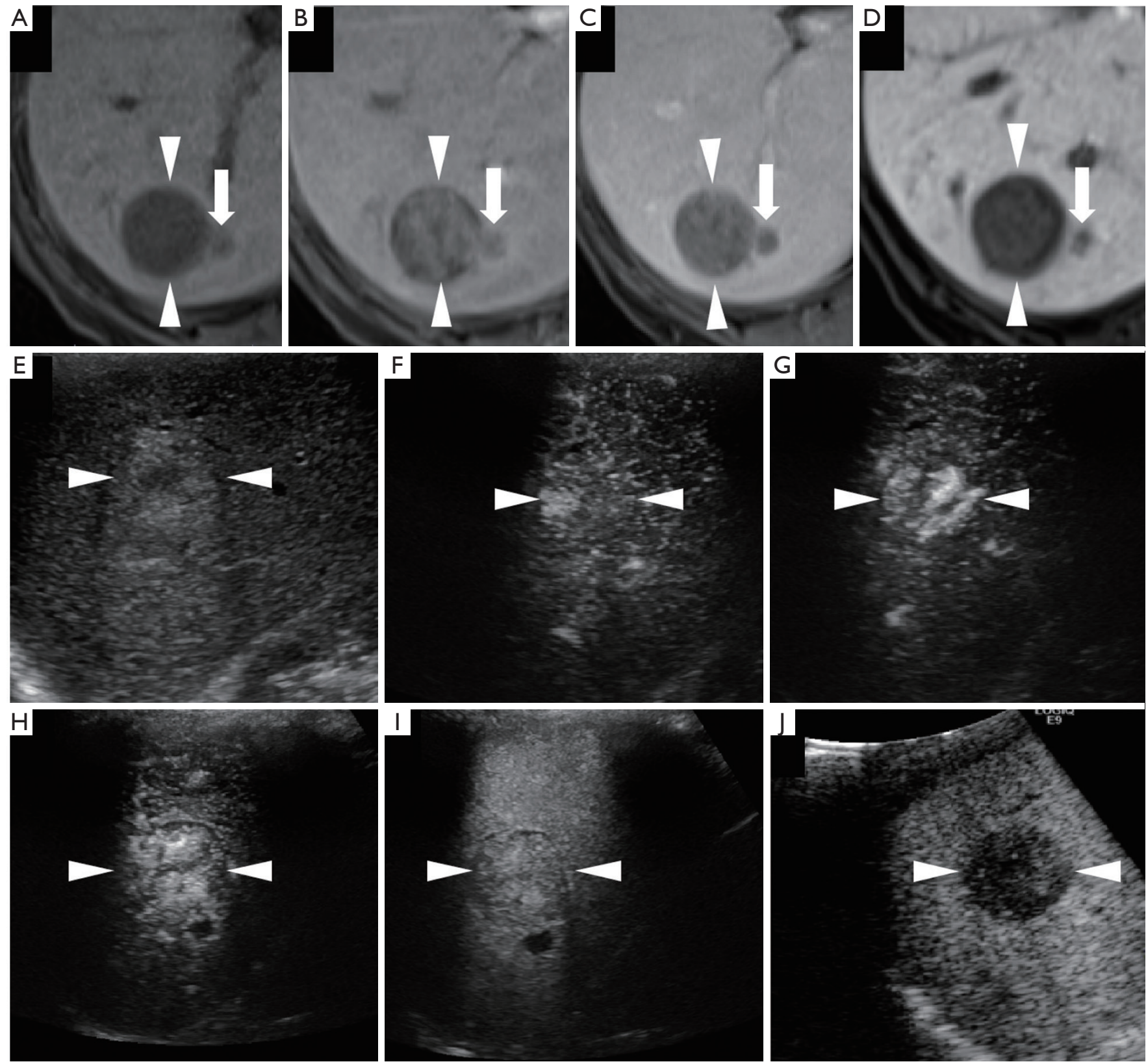

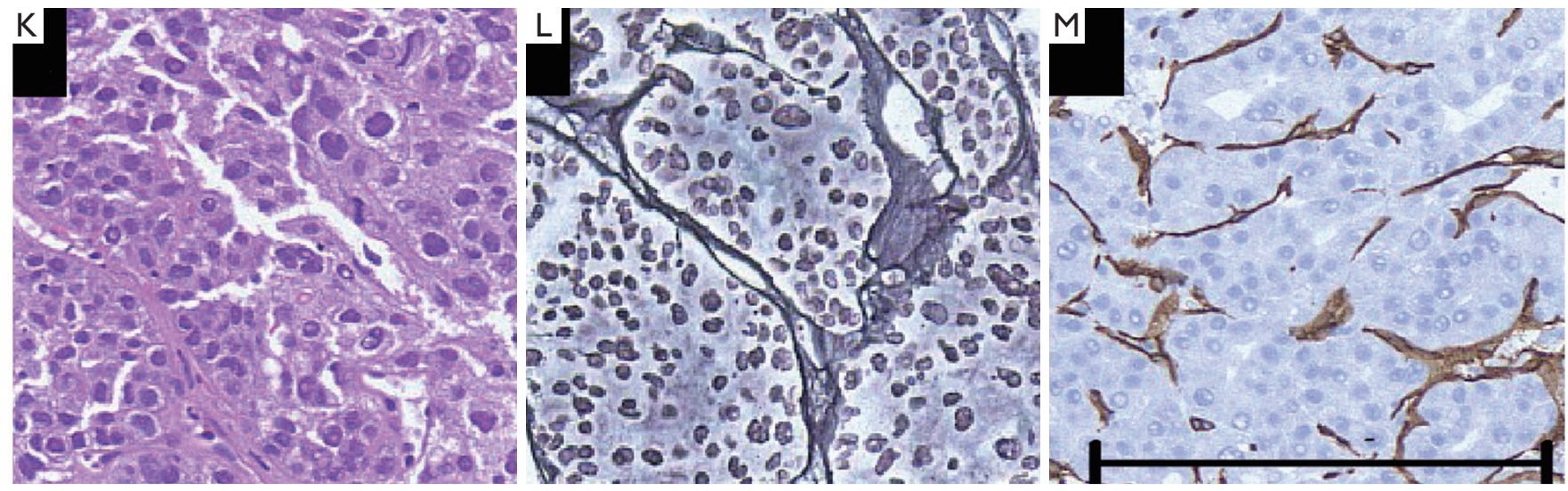

Figure 6 A non-hypervascular moderately differentiated hepatocellular carcinoma (HCC) lesion (maximum diameter, 30 mm) in segment VI/VII was found in a 76-year-old female patient with chronic hepatitis C. (A) Axial T1WI images on a plain scan show homogeneous hypointensity in the tumor, with a regular oval shape and distinct margin. The pre-contrast ratio was 0.60 . (B,C) On the arterial phase $(\mathrm{B}$, 25 seconds) and portal phase (C, 70 seconds) of gadolinium-ethoxybenzyl-diethylenetriamine pentaacetic acid magnetic resonance imaging (EOB-MRI), the lesion is shown as a hypointensity compared with adjacent liver parenchyma. (D) Twenty minutes after EOB agent injection, the lesion was clearly shown as a homogeneous hypointensity on the hepatobiliary phase of EOB-MRI. The post-contrast ratio was 0.403 and the EOB enhancement ratio was 0.670. (E) On the grayscale ultrasound (US), the targeted lesion appears as an ill-defined, heterogeneous, hyperechoic lesion, with a small hypoechoic area in the center. (F,G,H) Using low mechanical index contrast imaging, the arterial phase of Sonazoid contrast-enhanced ultrasound (SCEUS) shows a significant hypervascular area with centripetal vessels. (I) During the portal phase, the lesion appears as a heterogeneous isovascularity. (J) The post-vascular phase image shows a hypoechoic lesion (late washout). (K) Hematoxylin-eosin (HE) staining shows obvious cancer cell atypia and hypercellularity. Cancer cells are arranged disorderly. (L) Silver staining shows that reticular fibers are sparsely distributed and not very clear. (M) Diffuse expression of cluster of differentiation 34 suggests increased neovascularization, resulting from sinusoidal capillarization and formation of sinusoidal vascular endothelium in HCC. The arrowheads seen in (A,B,C,D,E,F,G,H,I,J) indicate the margin of the lesion. Arrows in (A,B,C,D) depict a small satellite lesion beside the lesion. The black bar length in the lower side of $(M)$ represents 200 microns $(\mu \mathrm{m})$ as a magnification reference for $(K, L, M)$. This lesion was pathologically diagnosed as a moderately differentiated HCC. (A,B,D,G,J) are quoted from (26).

66.7\%) and moderately diff. HCC (30/37, 81.0\%) decreased relative to that of eHCC. Poorly diff. HCCs receive their blood supply entirely from neovascularized unpaired arteries rather than from portal veins, and almost all venous drainage occurs through the portal veins or sinusoids rather than the hepatic veins $(28,29)$. The decline in blood supply results in a typical 'washout' appearance of the lesions in the PP of SCEUS. Accordingly, in our study, most of the poorly diff. HCC lesions $(22 / 31,71.0 \%)$ showed a hypovascular appearance in the PP.

Furthermore, for the PP, the hemodynamic change in poorly diff. HCC was the most significant (there were statistical differences between poorly diff. HCC and any other histological grades). In this regard, the PP may be more suitable for the diagnosis of poorly diff. HCC than for eHCC.

A few minutes after injection, the Sonazoid agent gradually infiltrates into the blood vessels' extracellular space. There, 99\% of the Sonazoid microbubbles are phagocytosed by Kupffer cells, providing characteristic Kupffer imaging in the PVP. Generally, eHCCs, which have as many Kupffer cells as surrounding non-cancerous liver cells, have an isoechoic pattern relative to noncancerous liver tissue in the PVP (30). Previous studies and our study found that the majority of eHCCs have an isoechoic appearance in the PVP. Korenaga et al. (14) found that $69.2 \%$ (9 of 13 lesions) of the well diff. HCCs (which correspond to eHCCs in our study) had an isoechoic pattern. In our study, the proportion was $85.9 \%$ (61 of 71 lesions). As HCC advances, the number of Kupffer cells in cancerous tissues decreases or even becomes zero (31). Korenaga et al. (14) also found that all of the moderately and poorly diff. HCCs in their study (which correspond to adHCCs in our study) showed a hypoechoic appearance. 

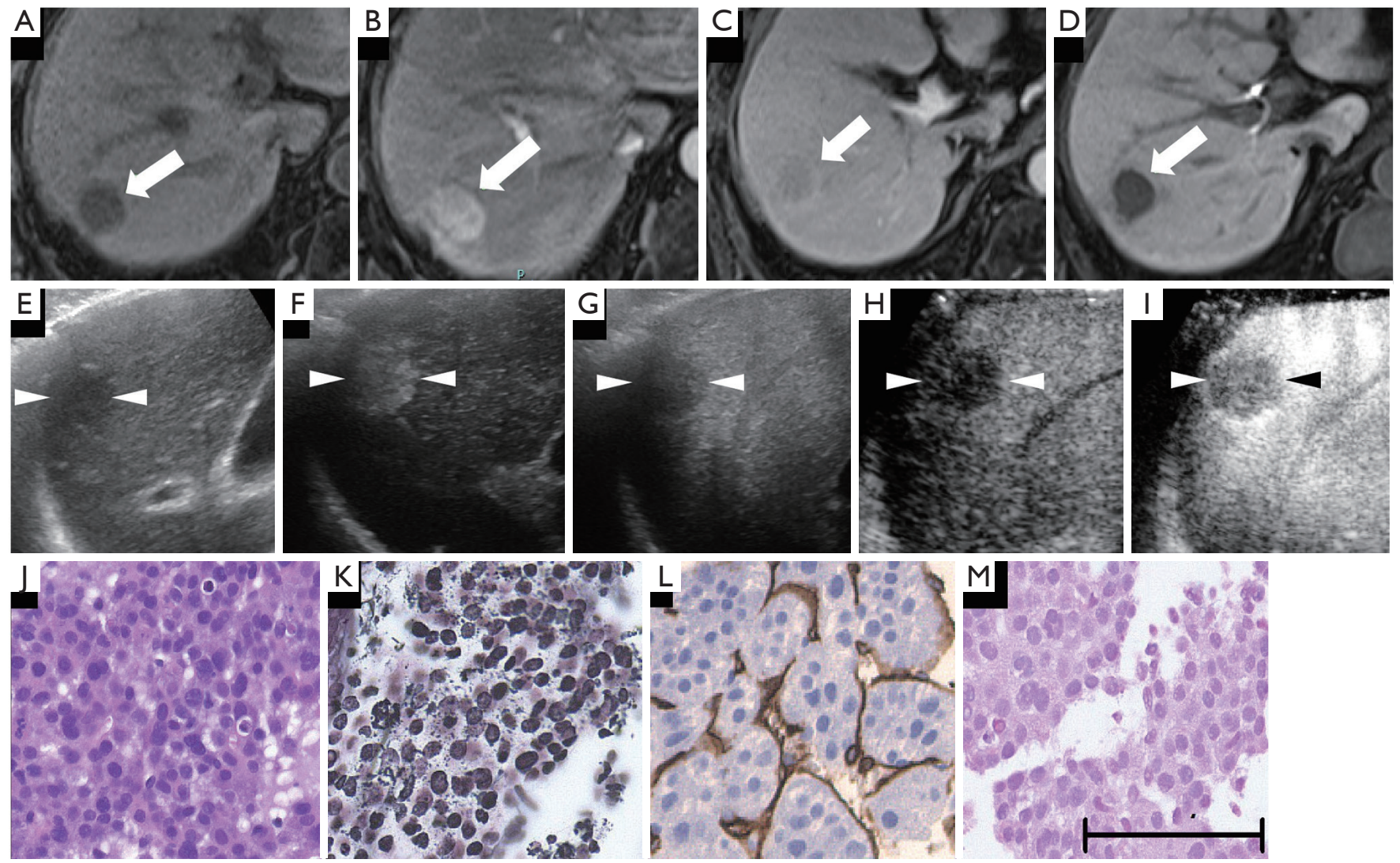

Figure 7 A hypervascular poorly differentiated hepatocellular carcinoma (HCC) lesion (maximum diameter, $23 \mathrm{~mm}$ ) in segment VII was found in a 76-year-old female patient with chronic hepatitis C. (A) Axial T1WI images on a plain scan show homogeneous hypointensity in the tumor, with a regular oval shape and distinct margin. The pre-contrast ratio was 0.62. (B) On the arterial phase of gadoliniumethoxybenzyl-diethylenetriamine pentaacetic acid magnetic resonance imaging (EOB-MRI) (25 seconds), the lesion is shown as a hyperintensity compared with the adjacent liver parenchyma. (C) On the portal phase (70 seconds) of EOB-MRI, the lesion is shown as a hypointensity compared with the adjacent liver parenchyma. (D) Twenty minutes after EOB agent injection, the lesion shows as a homogeneous hypointensity (arrow) on the hepatobiliary phase. The post-contrast ratio was 0.47 , and the EOB enhancement ratio was 0.75. (E) In the grayscale ultrasound (US), the targeted lesion appears as a well-defined, homogeneous, hyperechoic lesion. (F) Using low mechanical index contrast imaging, the arterial phase Sonazoid contrast-enhanced ultrasound (SCEUS) shows hypervascularity. (G) During the portal phase, the lesion shows hypovascularity (typical washout). (H) The post-vascular phase image shows a significant hypoechoic lesion. (I) After switching to high mechanical index contrast imaging, the post-vascular phase image shows extremely significant hypoechoicity. (J) Hematoxylin-eosin staining reveals a large, bizarre-shaped nucleus, with hypercellularity and an increased nuclear cytoplasmic ratio. (K) Silver staining shows that reticular fibers disappeared. (L) Positive immunoreactivity in sinusoidal capillarization, showing a diffuse cluster of differentiation 34 expressions. (M) Cytokeratin 7 staining shows no expression in tumor areas, resulting in no ductular reaction. The arrows seen in (A,B,C,D) and the arrowheads seen in (E,F,G,H,I) indicate the margin of the lesion. The black bar length in the lower side of $(\mathrm{M})$ represents 100 microns $(\mu \mathrm{m})$ as a magnification reference for $(\mathrm{J}, \mathrm{K}, \mathrm{L}, \mathrm{M})$. All the above histopathological findings were compatible with poorly differentiated HCC. (D,E,F,I) are quoted from (26).

Similarly, our study showed that most (well diff. HCC: 18/24, 75\%) or almost all (moderately diff. HCC: $36 / 37$, $97.3 \%$; poorly diff. HCC: $31 / 31,100 \%)$ of the adHCCs had a hypoechoic appearance in the PVP. Some researchers believe that "changes in tumor vascularity in PP" precede "microbubble contrast accumulation deficit in PVP" as HCC advances (32). From this perspective, there is a viewpoint that observation of the PP could be more 
Table 3 Comparison of radiological characteristics for $\mathrm{eHCC}^{\dagger \ddagger}$

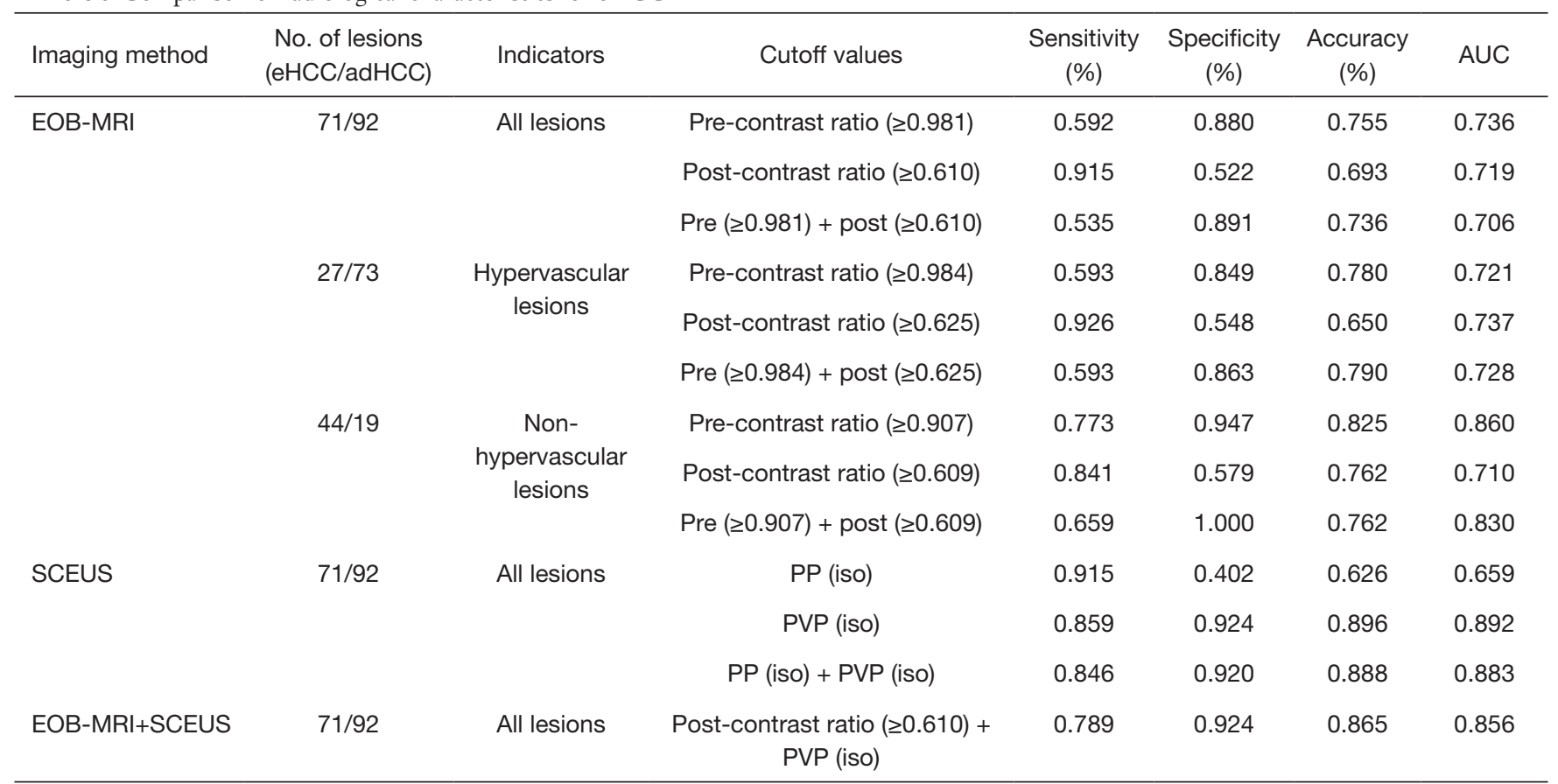

${ }^{\dagger}$, this diagnosis is made by differential eHCC from advanced HCC. Advanced HCC was defined as taking well, moderately, poorly diff. HCC as a whole. ₹, HCC, hepatocellular carcinoma; eHCC, early HCC; SCEUS, Sonazoid contrast enhanced ultrasound; EOB-MRI, gadolinium ethoxybenzyl diethylenetriamine pentaacetic acid magnetic resonance imaging; AP, arterial phase, PP, portal phase; PVP, postvascular phase; AUC, area under the curve.

Table 4 Comparison of EOB-MRI between hypervascular and non-hypervascular eHCC

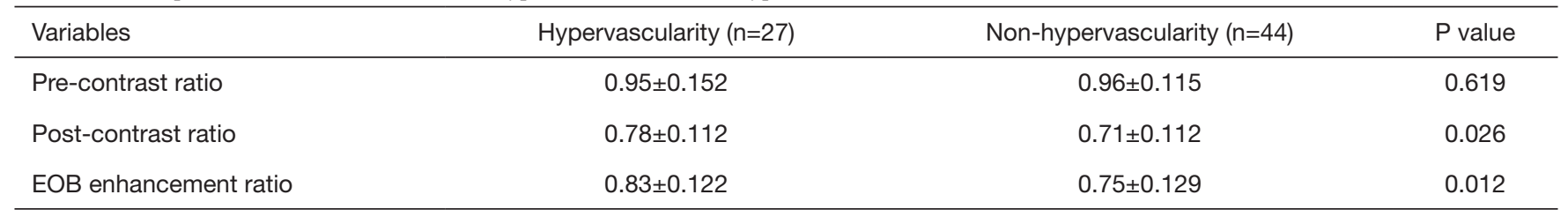

EOB-MRI, gadolinium ethoxybenzyl diethylenetriamine pentaacetic acid magnetic resonance imaging; eHCC, early hepatocellular carcinoma.

effective than that of PVP imaging for identifying the HCC histological grade (33). Contrastingly, our research observed that, compared with vascularity in the PP, echogenicity in the PVP had a more marked decreasing trend as HCC advanced (Table 2, the PP and PVP showed Spearman correlation indexes of 0.472 and 0.746 , respectively). Moreover, Sonazoid-specific PVP images demonstrated their strong ability to aid in eHCC diagnosis. In the current study, the determination of the isoechogenicity in the PVP yielded an accuracy of $89.6 \%$ and an AUC of 0.892 for eHCC diagnosis. Both values were higher than any other indicators and patterns. Taking these findings into account, we consider PVP to be more effective than PP in histological grade differentiation, especially in eHCC diagnosis.

The study of the HBP using EOB-MRI yielded promising results in the grade differential diagnosis of HCC. In our study, as the histological grading advanced, the post-contrast ratio showed a statistically decreasing trend (the Spearman correlation indexes for all lesions and hypervascular lesions were -0.488 and -0.417 , respectively). The newly developed hepatocyte-specific MR agent $\mathrm{EOB}$, which metabolizes in the AP and $\mathrm{PP}$, is similar to the commonly used iodine- and gadolinium-based agents, 
which are firstly distributed via the bloodstream in the AP, diffuse into the extravascular interstitium, and then exit tumor capillaries in the PP (10). EOB-MRI's specificity is that in the HBP, approximately $50 \%$ of the EOB agent can be taken up by functional hepatocytes via the organic anion transporter polypeptides (OATPs) (34), which are located on the basolateral membrane of hepatocytes. Previous research found that OATPs gradually decrease as HCC advances (35). Therefore, a change in OATPs can partly explain the working mechanism of the HBP in the histological grading diagnosis of HCC (36).

Furthermore, liver function may have some relationship with histological grading. Kim et al. (17) found that in patients with relatively poor liver function (Child-Pugh class B and C groups), there was no significant difference in the signal intensity ratio of the HBP among different histological grades. In contrast, there was a significant difference in patients with relatively normal liver function (Child-Pugh class A group) ( $\mathrm{P}=0.01-0.001)$. However, there is no direct, reliable evidence regarding whether liver function impacts histological grade.

According to our results, the PVP of SCEUS is superior to the HBP in EOB-MRI for histological grade differential diagnosis. We suspect that the complex secretion pathways of the EOB agent may play a role in decreasing the efficacy of the HBP. Sonazoid microbubbles are directly released unchanged from the liver back into circulation and then expired via the lungs (37). This direct metabolic pathway is rarely affected, except for in severe respiratory dysfunction. Contrastingly, the EOB agent is eliminated in a more complex non-metabolized form, excreted either into the biliary canaliculi via the multidrug resistance-associated proteins (MRP2) (43.1-53.2\%), or through renal glomerular filtration to urine (41.6-51.2\%) (34). In total, the majority of HCC lesions display a hypoenhanced appearance in the HBP. However, hyper- and isoenhancement were observed in $6-15 \%$ of HCC lesions (38), regardless of their histological grade (39). This paradoxical phenomenon can be explained by prominent bile secretion, impairment of bile excretion by compression effects of tumors, and high preservation of transportation systems such as MRP2 $(39,40)$. These factors may significantly decrease the contrast agent's excretion, thus promoting the accumulation of the EOB contrast agent in lesions. However, it is still uncertain as to whether and to what extent the MRP2 transport system, the biliary tract, and renal function affect HCC of different histological grades.

The average signal intensity ratio in unenhanced $\mathrm{T} 1$ - weighted images was found to decrease as HCC progresses both in our research (the Spearman correlation indexes for all lesions and for hypervascular lesions were -0.422 and -0.312 , respectively) and in the literature (41). In our study, $14.1 \%$ of eHCCs showed hyperenhancement on unenhanced T1-weighted images, higher than both moderately $(5.4 \%)$ and poorly (3.2\%) diff. HCC. On unenhanced T1-weighted images, most of the eHCCs were iso- (30 in 71 lesions) and hyperenhanced (31 in 71 lesions), while the adHCCs generally showed hypoenhancement (81 in 92 lesions). These phenomena may be due to the gradual changes in intratumoral components among different histological grades. In the early stages of HCC, the high intracellular content of certain substances, such as the rich cytoplasm, the accumulation of fat, intralesional glycogen and copper contents, and high protein content caused by active protein synthesis, are presumed to be closely related to hyperenhancement on unenhanced T1-weighted images (42). The hypoenhancement on T1-weighted images is due to the change in hypercellularity (specifically, there may be an increased cell density and small cell changes) and the intralesional hypervascular supply during hepatocellular carcinogenesis $(36,43)$. This change leads to an increased amount of water; thus, the intratumoral signal intensity is reduced accordingly $(36,43)$. As HCC progresses, the changes in hypercellularity and hypervascular supply are even more pronounced. Meanwhile, liquefactive necrosis, which frequently occurs in adHCC, is regarded as a key pathological feature of moderately and poorly diff. HCC (23), can also contribute to higher intracellular water content and reduce the intralesional signal intensity (36).

The diagnostic efficacy of imaging methods for tumor arterial vascular-related HCC was one of the big concerns in this study. During hepatocarcinogenesis, hypovascularity occurs due to insufficient neovascularization and impaired portal blood supply (29). In contrast, hypervascular lesions are mainly fed by the marked increase in neovascularized sinusoidal capillarization and unpaired hepatic arteries (44). These vascular differences have attractive importance. Firstly, arterial vascularity during hepatocarcinogenesis is closely related to the histological grading of HCC (45). Non-hypervascular HCC lesions generally occur in eHCC (Table 3, 44/63, 69.8\%). Simultaneously, most eHCC lesions are non-hypervascular lesions (Table 4, 44/71, $62.0 \%)(21)$. Secondly, non-hypervascular HCC shows a marginally lower incidence of microvascular invasion and metastases than hypervascular lesions (46), suggesting that it is biologically less aggressive. Thirdly, some prospective 
studies have indicated that non-hypervascular lesions gradually develop hypervascularization within the followup period $(46,47)$. Given these factors, non-hypervascular lesions warrant close attention, as they may act as early predictors of the multistep process of hepatocarcinogenesis. Our study revealed several positive tumoral vascular-related results. Typically, using EOB-MRI, non-hypervascular HCC yielded a relatively higher efficacy than hypervascular HCC (Table 3). In particular, the use of the HBP in EOBMRI showed strong diagnostic ability, with the highest sensitivity $(92.6 \%)$ for hypervascular lesions, while the combination of unenhanced T1-weighted images and HBP in EOB-MRI yielded perfect specificity (100\%) for nonhypervascular lesions.

Furthermore, the HBP in EOB-MRI could distinguish non-hypervascular eHCC from hypervascular eHCC (Table 4). Based on these promising results, our study highlighted the value of the HBP in EOB-MRI for predicting the vascularity of HCC, especially for nonhypervascular lesions. Unfortunately, as hyper- and nonhypervascular eHCCs have very similar signal intensity ratio values in unenhanced $\mathrm{T} 1$-weighted images $(\mathrm{P}=0.619)$ (Table 4), we speculate that the use of unenhanced T1weighted images is unfeasible for vascular identification of eHCCs. This negative result may be because unenhanced T1-weighted MRI is sensitive to detecting the change in composition rather than the vascularity of the lesion. Nevertheless, the explanations above must be verified through thorough and reliable data collection.

Our study found that in both non-hypervascular lesions and in all lesions, the unenhanced T1-weighted images had better diagnostic results for eHCC than HBP images or the combined application of unenhanced T1-weighted images and HBP images. Thus, we cannot conclude that unenhanced T1-weighted images can be used to diagnose eHCC independently. More than half of these lesions showed an iso- or hyperenhanced appearance (40/71, 56.3\%) on unenhanced T1-weighted images. If we independently use unenhanced T1-weighted images for the diagnosis of eHCC, the isoenhanced lesions will be missed, and the hyperenhanced lesions can be easily misdiagnosed as benign. Of the 163 lesions in this study, except for 1 poorly diff. HCC lesion (hyperenhancement), all other lesions were hypoenhanced in HBP images. In this regard, before performing histological classification diagnosis, HBP images are a good reference for undetectable lesions on unenhanced T1-weighted images and confirm the malignant characteristics of lesions.
There are some limitations to this study. Firstly, histological diagnosis of all HCC lesions was conducted with specimens using US-guided biopsy rather than by surgical resection or autopsy. As there is unsatisfactory heterogeneity in the microscopic assessment of this type of tumor, some researchers believe that the limited samples obtained by biopsy may hinder its full predictive value in HCC (48). Secondly, selection bias may not have been completely avoided due to the relatively small target lesions' size. We excluded 10 large lesions (50-200 mm). As previously described in the methods section, they could not be fully displayed on the SCEUS machine screen and/or had large complex internal components (necrosis or hemorrhage), which made it difficult to judge the vascularity and the degree of enhancement. In the present study, $87 \%$ (130/163) of the lesions enrolled were not larger than $20 \mathrm{~mm}$ in diameter and were defined as small HCCs or small-size HCCs $(33,49)$. These HCCs are regarded as having certain characteristics in terms of histology and vascularity (50), although they have not yet been fully explored. Thirdly, this study mainly introduced the value of non-invasive imaging modalities in the histological grading diagnosis of HCC. Unfortunately, due to time constraints and the manuscript's length, it did not involve the diagnosis of intrahepatic cholangiocellular carcinoma (ICC), which can be easily misdiagnosed as HCC. Some high-quality multicenter and prospective studies have confirmed the statistical differences in the appearance of ICC and HCC in different phases of SonoVue CEUS $(51,52)$. Inspired by these promising results, it is worth exploring whether SCEUS and EOB-MRI can perform differential diagnosis of HCC between different histological grades and ICC. Lastly, for non-hypervascular lesions, we found statistical differences in the target patterns in EOBMRI between eHCC and adHCC (Table 3). However, due to the small sample size of non-hypervascular adHCCs (2, 9, and 8 lesions were well, moderately, and poorly diff. HCC, respectively), we could not perform further statistical analysis of the diagnostic efficacy according to the detailed histological grading.

In summary, unenhanced T1-weighted images and the HBP in EOB-MRI (regardless of tumor vascularity in the AP), and the PP and PVP in SCEUS can be used as alternative imaging tools for the prediction of the histological grade of HCC. Given the relatively better performance of the PVP in terms of histological grade differentiation, we recommend that priority be given to applying the PVP in SCEUS, especially the isoechogenicity 
in the PVP for eHCC diagnosis. We suggest that appropriate cutoff values greater than 0.981 (contrast ratio on unenhanced T1-weighted images) and 0.610 (contrast ratio on HBP images) are used as the quantitative criteria for the histological grading diagnosis of eHCCs. This study of accurate histological diagnosis may help histologicalrelated clinical decision-making (e.g., treatment and prognosis) in HCC. Nevertheless, further large-sample, multicenter, prospective comparative studies are needed to confirm and expand our findings.

\section{Acknowledgments}

Funding: None.

\section{Footnote}

Conflicts of Interest: All authors have completed the ICMJE uniform disclosure form (available at http://dx.doi. org/10.21037/qims-20-685). The authors have no conflicts of interest to declare.

Ethical Statement: Our retrospective study design was approved by the institutional review board of Yokohama City University Medical Center (Number B180200054) and complied with the Declaration of Helsinki principles. The requirement for informed consent was waived.

Open Access Statement: This is an Open Access article distributed in accordance with the Creative Commons Attribution-NonCommercial-NoDerivs 4.0 International License (CC BY-NC-ND 4.0), which permits the noncommercial replication and distribution of the article with the strict proviso that no changes or edits are made and the original work is properly cited (including links to both the formal publication through the relevant DOI and the license). See: https://creativecommons.org/licenses/by-ncnd/4.0/.

\section{References}

1. Villanueva A. Hepatocellular carcinoma. N Engl J Med 2019;380:1450-62.

2. Njei B, Rotman Y, Ditah I, Lim JK. Emerging trends in hepatocellular carcinoma incidence and mortality. Hepatology 2015;61:191-9.

3. Farinati F, Sergio A, Baldan A, Giacomin A, Di Nolfo MA, Del PP, Benvegnu L, Rapaccini G, Zoli M, Borzio F,
Giannini EG, Caturelli E, Trevisani F. Early and very early hepatocellular carcinoma: When and how much do staging and choice of treatment really matter? A multi-center study. BMC Cancer 2009;9:33.

4. Yamamoto M, Takasaki K, Otsubo T, Katsuragawa H, Katagiri S, Yoshitoshi K, Ariizumi S, Saito A, Nakano $M$. Favorable surgical outcomes in patients with early hepatocellular carcinoma. Ann Surg 2004;239:395-99.

5. Tamura S, Kato T, Berho M, Misiakos EP, O'Brien C, Reddy KR, Nery JR, Burke GW, Schiff ER, Miller J, Tzakis AG. Impact of histological grade of hepatocellular carcinoma on the outcome of liver transplantation. Arch Surg 2001;136:25-30; discussion 31.

6. Inoue K, Takayama T, Higaki T, Watanabe Y, Makuuchi M. Clinical significance of early hepatocellular carcinoma. Liver Transpl 2004;10:S16-9.

7. Omata M, Cheng AL, Kokudo N, Kudo M, Lee JM, Jia J, Tateishi R, Han KH, Chawla YK, Shiina S, Jafri W, Payawal DA, Ohki T, Ogasawara S, Chen PJ, Lesmana C, Lesmana LA, Gani RA, Obi S, Dokmeci AK, Sarin SK. Asia-Pacific clinical practice guidelines on the management of hepatocellular carcinoma: A 2017 update. Hepatol Int 2017;11:317-70.

8. Cai MY, Wang FW, Li CP, Yan LX, Chen JW, Luo RZ, Yun JP, Zeng YX, Xie D. Prognostic factors affecting postoperative survival of patients with solitary small hepatocellular carcinoma. Chin J Cancer 2016;35:80.

9. Yoneda N, Matsui O, Kobayashi S, Kitao A, Kozaka K, Inoue D, Yoshida K, Minami T, Koda W, Gabata T. Current status of imaging biomarkers predicting the biological nature of hepatocellular carcinoma. Jpn J Radiol 2019;37:191-208.

10. Sagrini E, Renzulli M, Pecorelli A, Stefanini F, Piscaglia F. Imaging of liver tumors in patients with chronic liver disease. Current Radiology Reports 2014;2:56.

11. Yan F, Song Z, Du M, Klibanov AL. Ultrasound molecular imaging for differentiation of benign and malignant tumors in patients. Quant Imaging Med Surg 2018;8:1078-83.

12. Wang F, Numata K, Nihonmatsu H, Okada M, Maeda S. Application of new ultrasound techniques for focal liver lesions. J Med Ultrason (2001) 2020;47:215-37.

13. Kudo M, Matsui O, Izumi N, Iijima H, Kadoya $M$, Imai Y, Okusaka T, Miyayama S, Tsuchiya K, Ueshima K, Hiraoka A, Ikeda M, Ogasawara S, Yamashita T, Minami T, Yamakado K. JSH Consensus-Based clinical practice guidelines for the management of hepatocellular carcinoma: 2014 update by the liver cancer study group of 
japan. Liver Cancer 2014;3:458-68.

14. Korenaga K, Korenaga M, Furukawa M, Yamasaki T, Sakaida I. Usefulness of Sonazoid contrast-enhanced ultrasonography for hepatocellular carcinoma: Comparison with pathological diagnosis and superparamagnetic iron oxide magnetic resonance images. J Gastroenterol 2009;44:733-41.

15. Ohama H, Imai Y, Nakashima O, Kogita S, Takamura M, Hori M, Seki Y, Sawai Y, Igura T, Fukuda K, Makino Y, Morimoto O, Ohsawa M, Sakamoto M, Murakami T. Images of Sonazoid-enhanced ultrasonography in multistep hepatocarcinogenesis: Comparison with Gd-EOB-DTPAenhanced MRI. J Gastroenterol 2014;49:1081-93.

16. Kogita S, Imai Y, Okada M, Kim T, Onishi H, Takamura M, Fukuda K, Igura T, Sawai Y, Morimoto O, Hori M, Nagano H, Wakasa K, Hayashi N, Murakami T. Gd-EOB-DTPA-enhanced magnetic resonance images of hepatocellular carcinoma: Correlation with histological grading and portal blood flow. Eur Radiol 2010;20:2405-13.

17. Kim HY, Choi JY, Kim CW, Bae SH, Yoon SK, Lee YJ, Rha SE, You YK, Kim DG, Jung ES. Gadolinium ethoxybenzyl diethylenetriamine pentaacetic acidenhanced magnetic resonance imaging predicts the histological grade of hepatocellular carcinoma only in patients with Child-Pugh class a cirrhosis. Liver Transpl 2012;18:850-7.

18. Duisyenbi Z, Numata K, Nihonmatsu H, Fukuda H, Chuma M, Kondo M, Nozaki A, Tanaka K, Maeda S. Comparison between low mechanical index and high mechanical index contrast modes of Contrast-Enhanced ultrasonography: Evaluation of perfusion defects of hypervascular hepatocellular carcinomas during the PostVascular phase. J Ultrasound Med 2019;38:2329-38.

19. Numata K, Luo W, Morimoto M, Kondo M, Kunishi Y, Sasaki T, Nozaki A, Tanaka K. Contrast enhanced ultrasound of hepatocellular carcinoma. World J Radiol 2010;2:68-82.

20. Nishigori S, Numata K, Irie K, Fukuda H, Chuma M, Maeda S. Fusion imaging with contrast-enhanced ultrasonography for evaluating the early therapeutic efficacy of radiofrequency ablation for small hypervascular hepatocellular carcinomas with iso-echoic or unclear margins on conventional ultrasonography. J Med Ultrason (2001) 2018;45:405-15.

21. Sugimoto K, Kim SR, Imoto S, Tohyama M, Kim SK, Matsuoka T, Yano Y, Kudo M, Hayashi Y. Characteristics of Hypovascular versus Hypervascular Well-Differentiated
Hepatocellular Carcinoma Smaller than $2 \mathrm{~cm}$ - Focus on Tumor Size, Markers and Imaging Detectability. Dig Dis 2015;33:721-7.

22. Inoue T, Hyodo T, Korenaga K, Murakami T, Imai Y, Higaki A, Suda T, Takano T, Miyoshi K, Koda M, Tanaka H, Iijima H, Ochi H, Hirooka M, Numata K, Kudo M. Kupffer phase image of Sonazoid-enhanced US is useful in predicting a hypervascularization of non-hypervascular hypointense hepatic lesions detected on Gd-EOB-DTPAenhanced MRI: A multicenter retrospective study. J Gastroenterol 2016;51:144-52.

23. International Working Party. Terminology of nodular hepatocellular lesions. Hepatology 1995;22:983-93.

24. Edmondson HA, Steiner PE. Primary carcinoma of the liver: A study of 100 cases among 48,900 necropsies. Cancer 1954;7:462-503.

25. International Consensus Group for Hepatocellular NeoplasiaThe International Consensus Group for Hepatocellular Neoplasia. Pathologic diagnosis of early hepatocellular carcinoma: A report of the international consensus group for hepatocellular neoplasia. Hepatology 2009;49:658-64.

26. Wang F, Numata K, Nihonmatsu H, Chuma M, Moriya S, Nozaki A, Ogushi K, Fukuda H, Ruan L, Okada M, Luo W, Koizumi N, Nakano M, Otani M, Inayama Y, Maeda S. Intraprocedurally EOB-MRI/US fusion imaging focusing on hepatobiliary phase findings can help to reduce the recurrence of hepatocellular carcinoma after radiofrequency ablation. Int J Hyperthermia 2020;37:1149-58.

27. Chung YE, Kim KW. Contrast-enhanced ultrasonography: Advance and current status in abdominal imaging. Ultrasonography 2015;34:3-18.

28. Kitao A, Zen Y, Matsui O, Gabata T, Nakanuma Y. Hepatocarcinogenesis: Multistep changes of drainage vessels at CT during arterial portography and hepatic arteriography--radiologic-pathologic correlation. Radiology 2009;252:605-14.

29. Kim JH, Joo I, Lee JM. Atypical appearance of hepatocellular carcinoma and its mimickers: How to solve challenging cases using gadoxetic Acid-Enhanced liver magnetic resonance imaging. Korean J Radiol 2019;20:1019-41.

30. Mandai M, Koda M, Matono T, Nagahara T, Sugihara T, Ueki M, Ohyama K, Murawaki Y. Assessment of hepatocellular carcinoma by contrast-enhanced ultrasound with perfluorobutane microbubbles: Comparison with dynamic CT. Br J Radiol 2011;84:499-507. 
31. Liu K, He X, Lei XZ, Zhao LS, Tang H, Liu L, Lei BJ. Pathomorphological study on location and distribution of Kupffer cells in hepatocellular carcinoma. World J Gastroenterol 2003;9:1946-9.

32. Maruyama H, Takahashi $M$, Ishibashi $H$, Okabe $S$, Yoshikawa M, Yokosuka O. Changes in tumor vascularity precede microbubble contrast accumulation deficit in the process of dedifferentiation of hepatocellular carcinoma. Eur J Radiol 2010;75:e102-6.

33. Maruyama H, Sekimoto T, Yokosuka O. Role of contrast-enhanced ultrasonography with Sonazoid for hepatocellular carcinoma: Evidence from a 10-year experience. J Gastroenterol 2016;51:421-33.

34. Lee NK, Kim S, Lee JW, Lee SH, Kang DH, Kim GH, Seo HI. Biliary MR imaging with Gd-EOB-DTPA and its clinical applications. Radiographics 2009;29:1707-24.

35. Kitao A, Matsui O, Yoneda N, Kozaka K, Shinmura R, Koda W, Kobayashi S, Gabata T, Zen Y, Yamashita T, Kaneko S, Nakanuma Y. The uptake transporter OATP8 expression decreases during multistep hepatocarcinogenesis: Correlation with gadoxetic acid enhanced MR imaging. Eur Radiol 2011;21:2056-66.

36. Bartolozzi C, Battaglia V, Bozzi E. HCC diagnosis with liver-specific MRI--close to histopathology. Dig Dis 2009;27:125-30.

37. Li P, Hoppmann S, Du P, Li H, Evans PM, Moestue SA, Yu W, Dong F, Liu H, Liu L. Pharmacokinetics of perfluorobutane after Intra-Venous bolus injection of sonazoid in healthy chinese volunteers. Ultrasound Med Biol 2017;43:1031-9.

38. Kim JW, Lee CH, Shin Park Y, Lee J, Choi JW, Kim KA, Park CM. Tips of differentiation of focal hepatic nodules on hepatobiliary phase of Gd-EOB-DTPA-enhanced MRI. 2015: European Congress of Radiology; 2015.

39. Tsuboyama T, Onishi H, Kim T, Akita H, Hori M, Tatsumi M, Nakamoto A, Nagano H, Matsuura N, Wakasa K, Tomoda K. Hepatocellular carcinoma: Hepatocyteselective enhancement at gadoxetic acid-enhanced MR imaging--correlation with expression of sinusoidal and canalicular transporters and bile accumulation. Radiology 2010;255:824-33.

40. Lee SA, Lee CH, Jung WY, Lee J, Choi JW, Kim KA, Park CM. Paradoxical high signal intensity of hepatocellular carcinoma in the hepatobiliary phase of Gd-EOB-DTPA enhanced MRI: Initial experience. Magn Reson Imaging 2011;29:83-90.

41. Cho ES, Choi JY. MRI features of hepatocellular carcinoma related to biologic behavior. Korean J Radiol
2015;16:449-64.

42. Furlan A, Marin D, Bae KT, Lagalla R, Agnello F, Bazzocchi M, Brancatelli G. Focal liver lesions hyperintense on T1-weighted magnetic resonance images. Semin Ultrasound CT MR 2009;30:436-49.

43. Sun H, Song T. Hepatocellular carcinoma: Advances in diagnostic imaging. Drug Discov Ther 2015;9:310-8.

44. Matsui O, Kobayashi S, Sanada J, Kouda W, Ryu Y, Kozaka K, Kitao A, Nakamura K, Gabata T. Hepatocelluar nodules in liver cirrhosis: Hemodynamic evaluation (angiography-assisted CT) with special reference to multi-step hepatocarcinogenesis. Abdom Imaging 2011;36:264-72.

45. Asayama Y, Yoshimitsu K, Nishihara Y, Irie H, Aishima S, Taketomi A, Honda H. Arterial blood supply of hepatocellular carcinoma and histologic grading: Radiologic-pathologic correlation. AJR Am J Roentgenol 2008;190:W28-34.

46. Takayasu K, Arii S, Sakamoto M, Matsuyama Y, Kudo M, Ichida T, Nakashima O, Matsui O, Izumi N, Ku Y, Kokudo N, Makuuchi M. Clinical implication of hypovascular hepatocellular carcinoma studied in 4,474 patients with solitary tumour equal or less than $3 \mathrm{~cm}$. Liver Int 2013;33:762-70.

47. Ogasawara S, Chiba T, Motoyama T, Kanogawa N, Saito T, Shinozaki Y, Suzuki E, Ooka Y, Tawada A, Kato H, Okabe S, Kanai F, Yoshikawa M, Yokosuka O. Prognostic significance of concurrent hypovascular and hypervascular nodules in patients with hepatocellular carcinoma. PLoS One 2016;11:e0163119.

48. Martins-Filho SN, Paiva C, Azevedo RS, Alves V. Histological grading of hepatocellular Carcinoma-A systematic review of literature. Front Med (Lausanne) 2017;4:193.

49. European Association for the Study of the Liver; European Association for the Study of the Liver. EASL Clinical Practice Guidelines: Management of hepatocellular carcinoma. J Hepatol 2018;69:182-236.

50. Nakashima O, Sugihara S, Kage M, Kojiro M. Pathomorphologic characteristics of small hepatocellular carcinoma: A special reference to small hepatocellular carcinoma with indistinct margins. Hepatology 1995;22:101-5.

51. Wildner D, Bernatik T, Greis C, Seitz K, Neurath MF, Strobel D. CEUS in hepatocellular carcinoma and intrahepatic cholangiocellular carcinoma in 320 patients - early or late washout matters: A subanalysis of the DEGUM multicenter trial. Ultraschall Med 
2015;36:132-9.

52. Wildner D, Pfeifer L, Goertz RS, Bernatik T, Sturm J, Neurath MF, Strobel D. Dynamic contrast-enhanced ultrasound (DCE-US) for the characterization of hepatocellular carcinoma and cholangiocellular carcinoma. Ultraschall Med 2014;35:522-7.

Cite this article as: Wang F, Numata K, Okada M, Chuma M, Nihonmatsu H, Moriya S, Nozaki A, Ogushi K, Luo W, Ruan L, Nakano M, Otani M, Inayama Y, Maeda S. Comparison of Sonazoid contrast-enhanced ultrasound and gadoliniumethoxybenzyl-diethylenetriamine pentaacetic acid MRI for the histological diagnosis of hepatocellular carcinoma. Quant Imaging Med Surg 2021;11(6):2521-2540. doi: 10.21037/qims20-685 